\title{
Unexpected reactivity of the Burgess reagent with thiols: Synthesis of symmetrical disulfides.
}

\author{
Scott C. Banfield, Alvaro Takeo Omori, Hannes Leisch, and Tomas Hudlicky* \\ Department of Chemistry and Center for Biotechnology, Brock University, 500 Glenridge Ave.
}

St. Catharines, Canada, L2S 3A1

TABLE OF CONTENTS

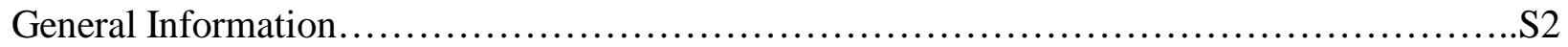

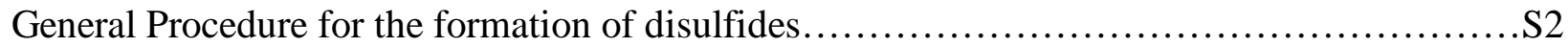

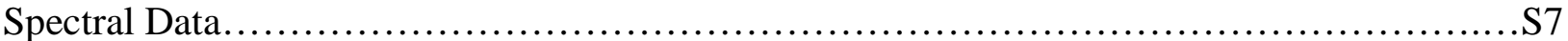




\section{General Experimental Methods}

All reactions were done in an argon atmosphere using standard Schlenk techniques for the exclusion of moisture and air. Liquid reagents were distilled prior to use, while other commercial solids were used as supplied. NMR analyses were performed in $\mathrm{CDCl}_{3}$; chemical shifts are quoted in ppm relative to TMS (as referenced to residual $\mathrm{CHCl}_{3} \delta_{H}=7.26$ or $\mathrm{CDCl}_{3} \delta_{\mathrm{C}}=77.0$ ) with coupling constants quoted in Hz. GC/MS was performed with $2 \mathrm{ml} / \mathrm{min}$ helium flow and following temperature program was used: $50{ }^{\circ} \mathrm{C}(2 \mathrm{~min}), 15^{\circ} \mathrm{C} / \mathrm{min}$ to $300{ }^{\circ} \mathrm{C}(3 \mathrm{~min})$.

\section{General procedure for the formation of disulfides:}

To a solution of Burgess reagent (1.05 eq.) in benzene (1 molar) was added dropwise the corresponding thiol (1 equiv) dissolved in benzene at rt. The progress of the reaction was followed by GC/MS. After complete conversion of the starting material (approximately 30 minutes to 1 hour) the reaction mixture was filtered through a plug of silica (hexanes) The crude product was either triturated with hexanes or purified by flash column chromatography.

\section{Didecyl disulfide (2) CAS: [10496-18-1]}

Following the general procedure using decanethiol $(0.59 \mathrm{ml}, 2.86 \mathrm{mmol})$ as starting material gave 0.47 $\mathrm{g}$ of didecyl disulfide $(95 \%) .{ }^{1} \mathrm{H}$ NMR $\left(300 \mathrm{MHz}, \mathrm{CDCl}_{3}\right) \delta=2.70(\mathrm{t}, J=7.2 \mathrm{~Hz}, 4 \mathrm{H}), 1.78-1.64(\mathrm{~m}$, $4 \mathrm{H}), 1.42-1.29(\mathrm{~m}, 28 \mathrm{H}), 0.90(\mathrm{t}, J=6.8 \mathrm{~Hz}, 6 \mathrm{H}) ;{ }^{13} \mathrm{C} \mathrm{NMR}\left(75 \mathrm{MHz}, \mathrm{CDCl}_{3}\right) \delta=39.2,31.9,29.6$, 29.5, 29.3, 29.2, 28.8, 28.5, 22.7, 14.4; LRMS (EI) $m / z 347(\mathrm{M}+1,25.4), 346\left(\mathrm{M}^{+}, 87.5\right), 278$ (18.9), 206 (14.0), 173 (10.0), 140 (14.3), 139 (37.7), 125 (11.0), 101 (11.1), 97 (20.1), 96 (13.2), 95 (11.1), 91 (34.7), 87 (20.5), 85 (48.3), 84 (10.3), 83 (21.2), 71 (48.7), 70 (24.2), 69 (34.4), 68 (10.2), 67 
(11.7), 60 (11.4), 57 (97.8), 56 (19.9), 55 (60.9), 47 (10.4), 45 (13.1), 43 (100.0), 42 (12.1), 41 (49.9);

HRMS (EI) calcd for $\mathrm{C}_{20} \mathrm{H}_{42} \mathrm{~S}_{2}: 346.2728$, found: 346.2727.

\section{bis(4-Chlorophenyl) disulfide (4) CAS: [1142-19-4]}

Following the general procedure using 4-chlorobenzenelthiol (0.207 g, $1.43 \mathrm{mmol})$ as starting material gave $0.190 \mathrm{~g}$ of bis(4-chlorophenyl) disulfide $(93 \%) .{ }^{1} \mathrm{H}$ NMR $\left(300 \mathrm{MHz}, \mathrm{CDCl}_{3}\right) \delta=7.45-7.38(\mathrm{~m}$, 4H), 7.30-7.24 (m, 4H); ${ }^{13} \mathrm{C}$ NMR $\left(75 \mathrm{MHz}, \mathrm{CDCl}_{3}\right) \delta=135.1,134.8,134.5,129.2 ; \mathrm{MS}(\mathrm{EI}) \mathrm{m} / z 288$ (M+1, 37.5), $287\left(\mathrm{M}^{+}, 7.5\right), 286$ (51.5), 145 (37.2), 144 (17.2), 143 (100.0), 108 (59.2), 99 (15.7), 84 (13.5), 75 (11.0), 73 (10.0), 69 (15.8), 63 (16.8); HRMS (EI) calcd for $\mathrm{C}_{12} \mathrm{H}_{8} \mathrm{Cl}_{2} \mathrm{~S}_{2}$ : 285.9444, found: 285.9444.

\section{bis(2-Phenylethyl) disulfide (6) CAS: [27846-22-6]}

Following the general procedure using phenylethylthiol $(0.192 \mathrm{ml}, 1.43 \mathrm{mmol})$ as starting material gave $0.181 \mathrm{~g}$ of bis(2-phenylethyl) disulfide $(92 \%) .{ }^{1} \mathrm{H}$ NMR $\left(300 \mathrm{MHz}, \mathrm{CDCl}_{3}\right) \delta=7.37-7.32(\mathrm{~m}, 4 \mathrm{H})$, 7.28-7.23 (m, 6H), 3.21-3.09 (m, 4H), 3.07-2.95 (m, 4H); ${ }^{13} \mathrm{C}$ NMR $\left(75 \mathrm{MHz}, \mathrm{CDCl}_{3}\right) \delta=140.0$, 139.8, 128.6 (2 overlapping signals), 128.5 (2 overlapping signals), 126.5, 126.4, 40.2, 39.9, 35.7, 35.3; LRMS (EI) m/z $275(\mathrm{M}+1,2.5), 274\left(\mathrm{M}^{+}, 12.3\right), 105$ (100.0), 77 (15.0); HRMS (EI) calcd for $\mathrm{C}_{16} \mathrm{H}_{18} \mathrm{~S}_{2}: 274.0850$, found 274.0854 .

\section{di-2-Naphthalenyl disulfide (8) CAS: [5586-15-2]}

Following the general procedure using 2-naphtalenethiol $(0.229 \mathrm{~g}, 1.43 \mathrm{mmol})$ as starting material gave $0.197 \mathrm{~g}$ of di-2-naphthalenyl disulfide $(90 \%)$. mp 135-138 ${ }^{\circ} \mathrm{C}$ (Ethyl Acetate; lit $\left.^{13}: 137-138{ }^{\circ} \mathrm{C}\right) ;{ }^{1} \mathrm{H}$ 
NMR (300 MHZ, $\left.\mathrm{CDCl}_{3}\right) \delta 7.96(\mathrm{~s}, 2 \mathrm{H}), 7.8-7.4(\mathrm{~m}, 12 \mathrm{H}) ;{ }^{13} \mathrm{C} \mathrm{NMR}\left(75 \mathrm{MHz}, \mathrm{CDCl}_{3}\right) \delta 134.3,133.5$, 132.5, 129.0, 127.8, 127.5, 126.7, 126.6, 126.2, 125.7; MS (EI) m/z (\%): 318 (100), 285 (3), 254 (8), 160 (41), 115 (43), 79 (10), 69 (9). HRMS (EI) calcd for $\mathrm{C}_{20} \mathrm{H}_{14} \mathrm{~S}_{2}$ 318.0537, found 318.0532

bis(tert-butyl) disulfide (10) CAS: [110-06-5] and bis(tert-butyl) trisulfide (11) CAS: [4253-90-1]

Following the general procedure using tert-butylthiol $(0.130 \mathrm{~g}, 1.43 \mathrm{mmol})$ as starting material gave $0.050 \mathrm{~g}(39 \%)$ of a mixture of di-tertbutyl disulfide and di-tertbutyl trisulfide in ratio of 3 to $1 .{ }^{1} \mathrm{H}$ NMR (300 MHZ, $\left.\mathrm{CDCl}_{3}\right) \delta 1.31(\mathrm{~s}, 9 \mathrm{H}) .{ }^{13} \mathrm{C} \mathrm{NMR}\left(75 \mathrm{MHz}, \mathrm{CDCl}_{3}\right) \delta 46.2,30.6$.

Diphenyl disulfide (13) CAS: [882-33-7]

Following the general procedure using benzenethiol $(0.158 \mathrm{~g}, 1.43 \mathrm{mmol})$ as starting material gave $0.150 \mathrm{~g}$ of diphenyl disulfide $(96 \%)$. mp 56-58 ${ }^{\circ} \mathrm{C}\left(\mathrm{CH}_{2} \mathrm{Cl}_{2}\right)\left(1 \mathrm{it}{ }^{14}: 58-60{ }^{\circ} \mathrm{C}\right.$, ethanol); ${ }^{1} \mathrm{H}$ NMR $(300$ $\left.\mathrm{MHZ}, \mathrm{CDCl}_{3}\right) \delta .7 .58-7.48(\mathrm{~m}, 4 \mathrm{H}), 7.34-7.21(\mathrm{~m}, 6 \mathrm{H}) ;{ }^{13} \mathrm{C} \mathrm{NMR}\left(75 \mathrm{MHz}, \mathrm{CDCl}_{3}\right) \delta 137.0,129.0$, 127.5, 127.1. MS (EI) m/z (\%): 218 (100), 185 (6), 154 (8), 141 (12), 109 (96), 77 (12), 65 (30), 51 (12). HRMS (EI) calcd for $\mathrm{C}_{12} \mathrm{H}_{10} \mathrm{~S}_{2} 218.0224$, found 218.0222

bis(4-Bromophenyl) disulfide (15) CAS: [5335-84-2]

Following the general procedure using 4-bromobenzenethiol $(0.300 \mathrm{~g}, 1.59 \mathrm{mmol})$ as starting material gave $0.287 \mathrm{~g}$ of bis(4-bromophenyl) disulfide $(95 \%)$ as white needles after crystallization from $\mathrm{CHCl}_{3}$. mp $94-95{ }^{\circ} \mathrm{C}\left(\mathrm{CHCl}_{3}\right) ;{ }^{1} \mathrm{H} \mathrm{NMR}\left(300 \mathrm{MHz}, \mathrm{CDCl}_{3}\right) \delta=7.45(\mathrm{~d}, J=8.4 \mathrm{~Hz}, 4 \mathrm{H}), 7.36(\mathrm{~d}, J=8.4 \mathrm{~Hz}$, $4 \mathrm{H}) ;{ }^{13} \mathrm{C} \mathrm{NMR}\left(75 \mathrm{MHz}, \mathrm{CDCl}_{3}\right) \delta=135.8,132.2,129.4,121.6 ; \mathrm{MS} \mathrm{m} / z 378(57), 377$ (15), 376 
(100), 374 (49), 190 (27), 189 (62), 188 (27), 187 (62), 140 (11), 109 (38), 108 (83), 82 (15), 69 (20),

63 (17); HRMS (EI) calcd for $\mathrm{C}_{12} \mathrm{H}_{8} \mathrm{Br}_{2} \mathrm{~S}_{2}$ 373.8434, found 373.8432 .

bis(Isopropyl) disulfide (17) CAS: [4253-89-8] and bis(Isopropyl) trisulfide (18) CAS: [5943-34-0]

Following the general procedure using 2-propanethiol $(0.200 \mathrm{~g}, 2.63 \mathrm{mmol})$ as starting material gave $0.179 \mathrm{~g}(85 \%)$ of a mixture of di-isopropyl disulfide and di-isopropyl trisulfide in ratio of 2 to 1 as colourless oil. ${ }^{1} \mathrm{H}$ NMR (300 MHz, $\left.\mathrm{CDCl}_{3}\right) \delta=3.21$ (sept, $J=6.6 \mathrm{~Hz}, 2 \mathrm{H}$, trisulfide), 2.97 (sept, $J=$ $6.6 \mathrm{~Hz}, 2 \mathrm{H}$, disulfide), $1.37\left(\mathrm{~d}, J=6.6 \mathrm{~Hz}, 6 \mathrm{H}\right.$, trisulfide), $1.30\left(\mathrm{~d}, J=6.6 \mathrm{~Hz}, 6 \mathrm{H}\right.$, disulfide); ${ }^{13} \mathrm{C}$ $\operatorname{NMR}\left(75 \mathrm{MHz}, \mathrm{CDCl}_{3}\right.$ ) $\delta=42.1$ (trisulfide), 41.5 (disulfide), 22.8 (trisulfide), 22.6 (disulfide); MS

(EI) m/z 182 (17), 150 (27), 108 (29), 98 (10), 75 (12), 43 (100), 41 (21); HRMS (EI) calcd for $\mathrm{C}_{6} \mathrm{H}_{14} \mathrm{~S}_{2}: 150.0537$, found 150.0539; HRMS (EI) calcd for $\mathrm{C}_{6} \mathrm{H}_{14} \mathrm{~S}_{3}: 182.0258$, found 182.0254 .

bis(4-Methoxyphenyl) disulfide (20) CAS: [5335-87-5]

Following the general procedure using 4-methoxybenzenethiol $(0.176 \mathrm{ml}, 1.43 \mathrm{mmol})$ as starting material gave $0.180 \mathrm{~g}$ of bis(4-methoxyphenyl) disulfide $(90 \%) .{ }^{1} \mathrm{H} \mathrm{NMR}\left(300 \mathrm{MHz}, \mathrm{CDCl}_{3}\right) \delta=7.43$ $7.37(\mathrm{~m}, 4 \mathrm{H}), 6.86-6.81(\mathrm{~m}, 4 \mathrm{H}), 3.79(\mathrm{~s}, 6 \mathrm{H}) ;{ }^{13} \mathrm{C} \mathrm{NMR}\left(75 \mathrm{MHz}, \mathrm{CDCl}_{3}\right) \delta=159.8,132.5,128.3$, 114.5, 55.3; LRMS (EI) m/z 279 (M+1, 9.8), $278\left(\mathrm{M}^{+}, 51.7\right), 140$ (25.4), 139 (100.0), 125 (12.1), 96 (12.4), 91 (14.3), ; HRMS (EI) calcd for $\mathrm{C}_{14} \mathrm{H}_{14} \mathrm{O}_{2} \mathrm{~S}_{2}$ : 278.0435, found: 278.0437.

bis(Phenylmethyl) disulfide (22) CAS: [150-60-7]

Following the general procedure using benzenemethanthiol $(0.169 \mathrm{ml}, 1.43 \mathrm{mmol})$ as starting material gave $0.164 \mathrm{~g}$ of bis(phenylmethyl) disulfide $(93 \%) .{ }^{1} \mathrm{H} \mathrm{NMR}\left(300 \mathrm{MHz}, \mathrm{CDCl}_{3}\right) \delta=7.41-7.34(\mathrm{~m}$, 
6H), 7.33-7.29 (m, 4H), $3.66(\mathrm{~s}, 4 \mathrm{H}),{ }^{13} \mathrm{C} \mathrm{NMR}\left(75 \mathrm{MHz}, \mathrm{CDCl}_{3}\right) \delta=137.3,129.4,128.4,127.4,43.2$;

LRMS (EI) $m / z 247(\mathrm{M}+1,3.0), 246\left(\mathrm{M}^{+}, 13.9\right), 91$ (100.0); HRMS (EI) calcd for $\mathrm{C}_{14} \mathrm{H}_{14} \mathrm{~S}_{2}: 246.0537$, found: 246.0541 .

\section{Sulfo-methylester carbamic acid triethlylammonium salt (31)}

Compound $\mathbf{3 1}$ was isolated by diluting the crude reaction mixture with diethyl ether, after following the general procedure. A white precipitate was formed which was filtered and dried under reduced pressure. mp 93 - $95{ }^{\circ} \mathrm{C}\left(\mathrm{Et}_{2} \mathrm{O}\right)$; IR (film) 3483, 3237, 2989, 2711, 2499, 1723, 1647, 1476, 1421, 1341, 1225, $1044,948,838 \mathrm{~cm}^{-1} ;{ }^{1} \mathrm{H}$ NMR $\left(300 \mathrm{MHz}, \mathrm{CDCl}_{3}\right) \delta=9.20(\mathrm{bs}, 1 \mathrm{H}), 7.15(\mathrm{bs}, 1 \mathrm{H}), 3.69(\mathrm{~s}, 3 \mathrm{H}), 3.15-$ $3.31(\mathrm{~m}, 6 \mathrm{H}), 1.39(\mathrm{tr}, J=7.2 \mathrm{~Hz}, 9 \mathrm{H}) ;{ }^{13} \mathrm{C}$ NMR $\left(75 \mathrm{MHz}, \mathrm{CDCl}_{3}\right) \delta=153.7,52.2,46.4,8.4$; HRMS (FAB) calcd for $\left(\mathrm{C}_{2} \mathrm{H}_{5} \mathrm{NO}_{5} \mathrm{~S} \cdot 2 \cdot \mathrm{C}_{6} \mathrm{H}_{15} \mathrm{~N}+\mathrm{H}\right)^{+}: 358.2376$, found: 358.2379 ; Anal calcd. for $\mathrm{C}_{2} \mathrm{H}_{5} \mathrm{NO}_{5} \mathrm{~S}$ $\cdot \mathrm{C}_{6} \mathrm{H}_{15} \mathrm{~N}$ : C 37.49, H 7.86, N 10.96; found : C 37.67, H 7.83, N 10.76. 

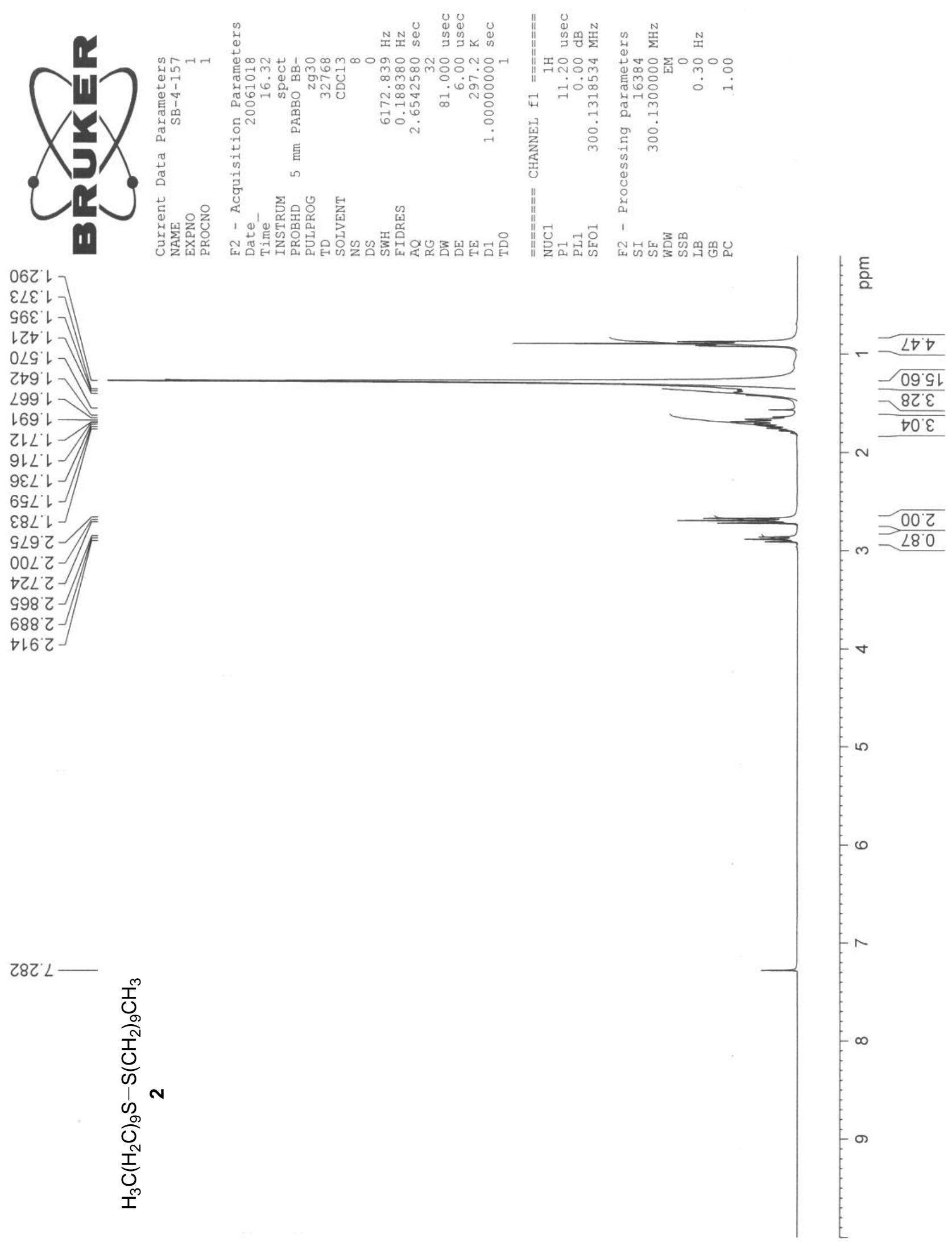


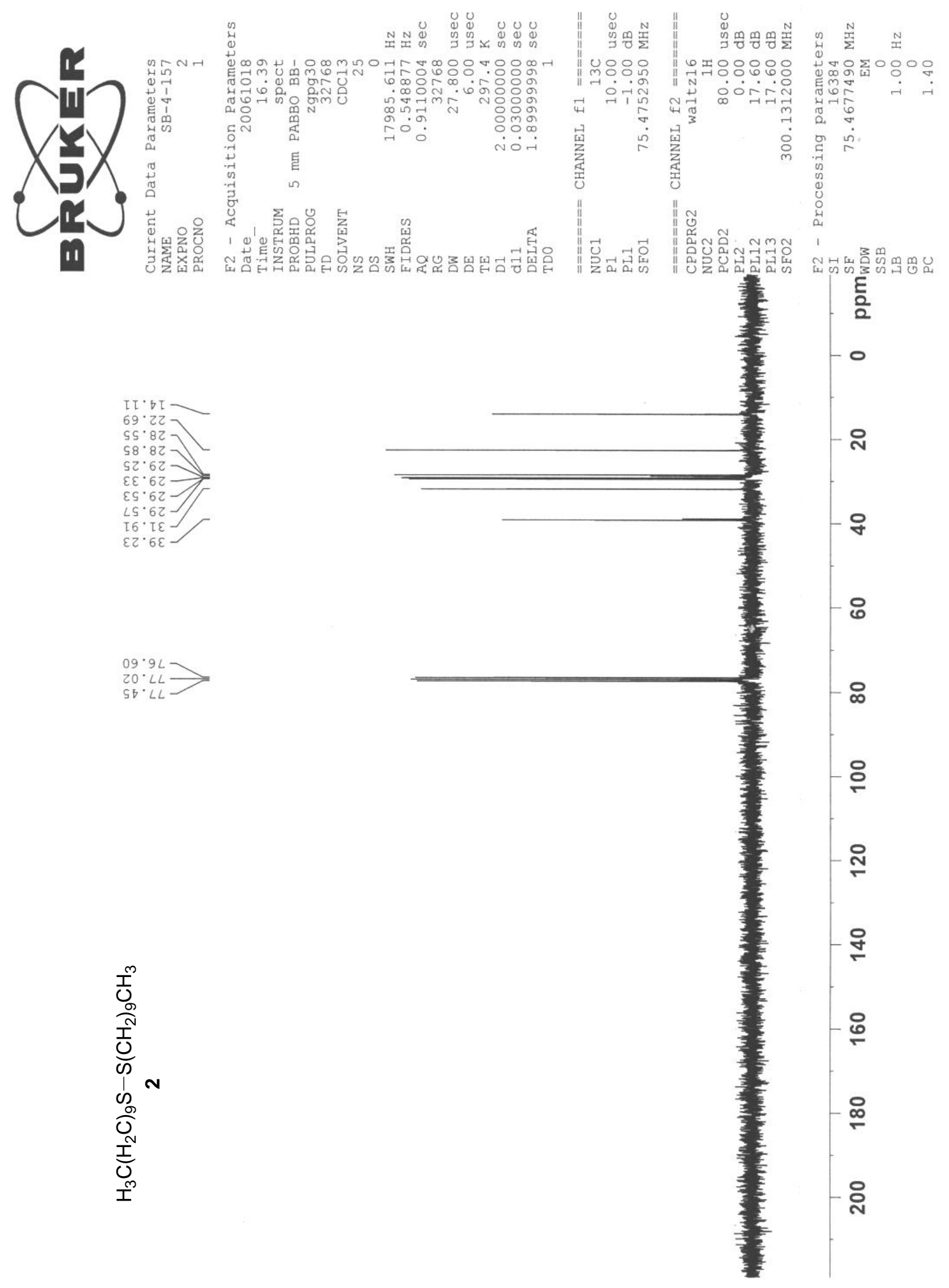



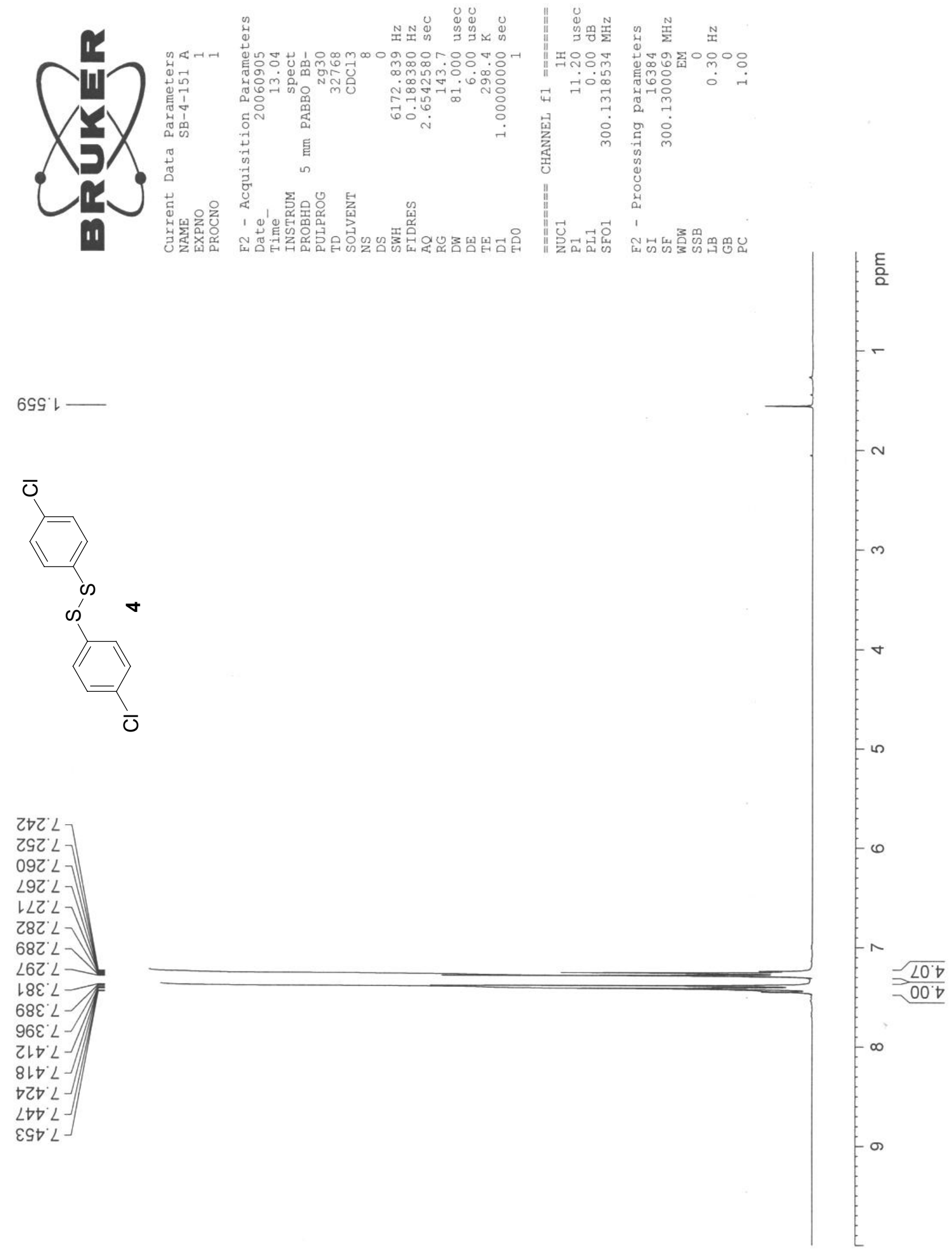


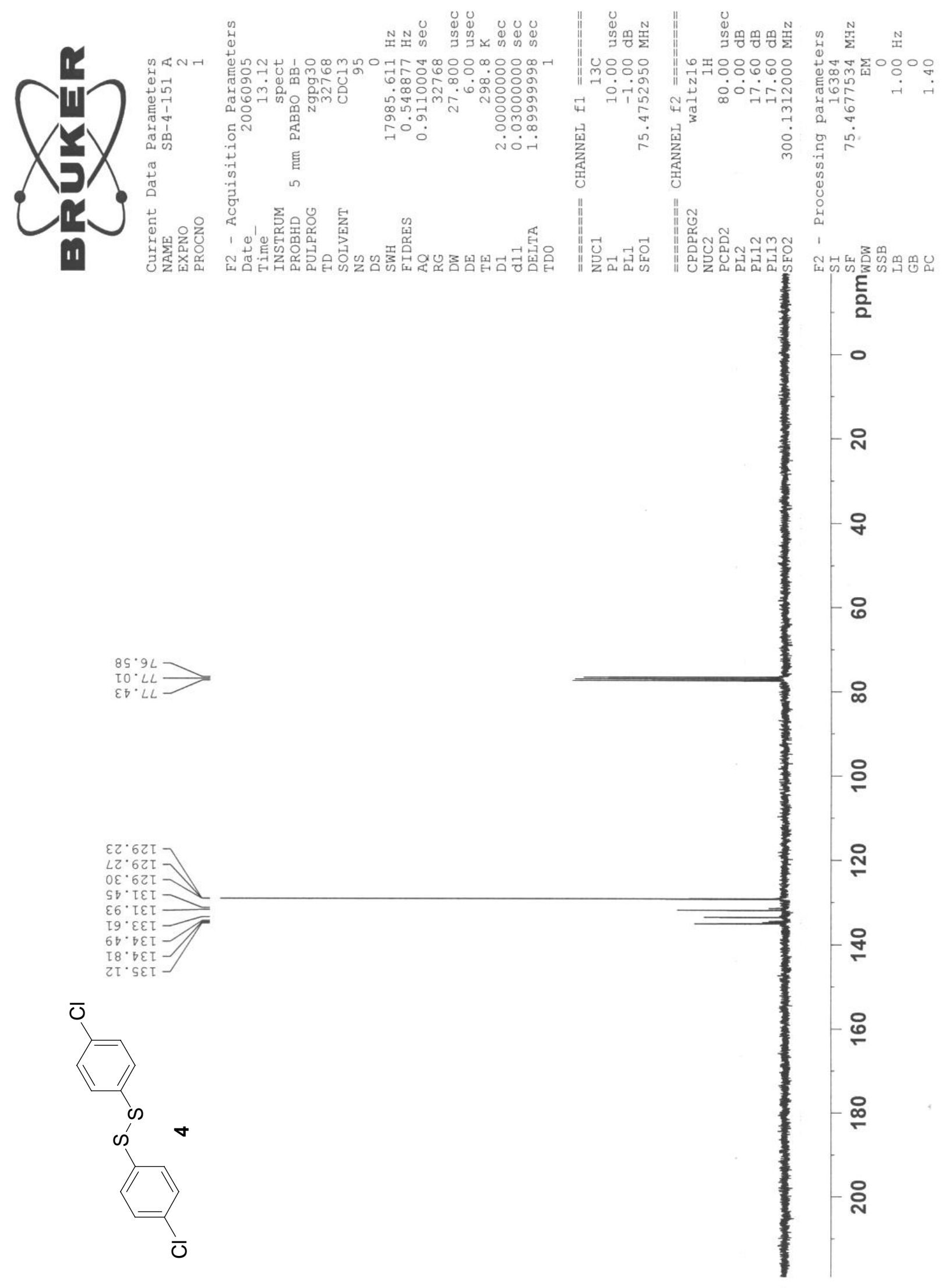



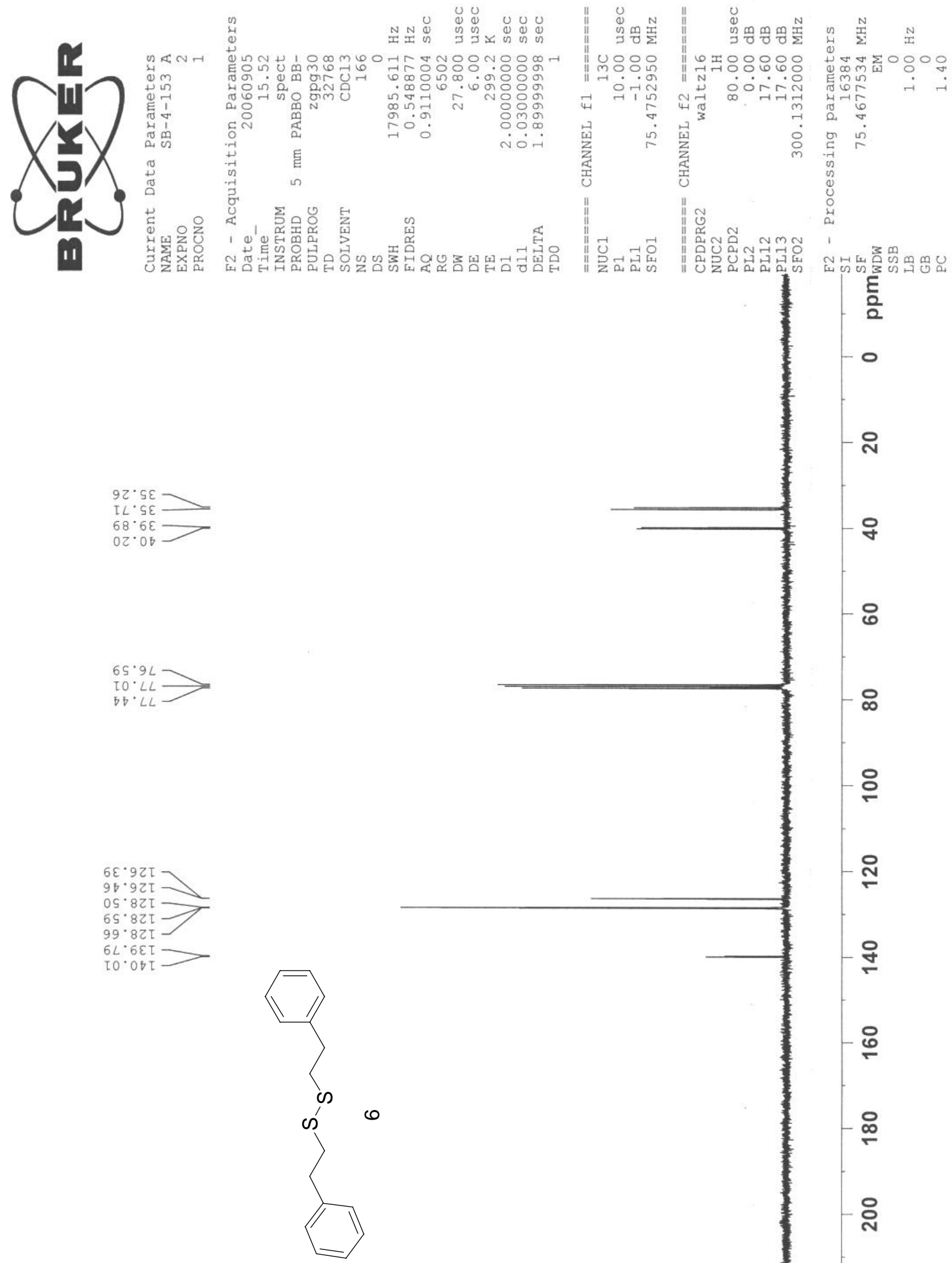

흘

ํ

q

웅

$\infty$

음

ิㅗำ

움

$\stackrel{\circ}{-}$

$\stackrel{\infty}{\circ}$

ㅇํ 

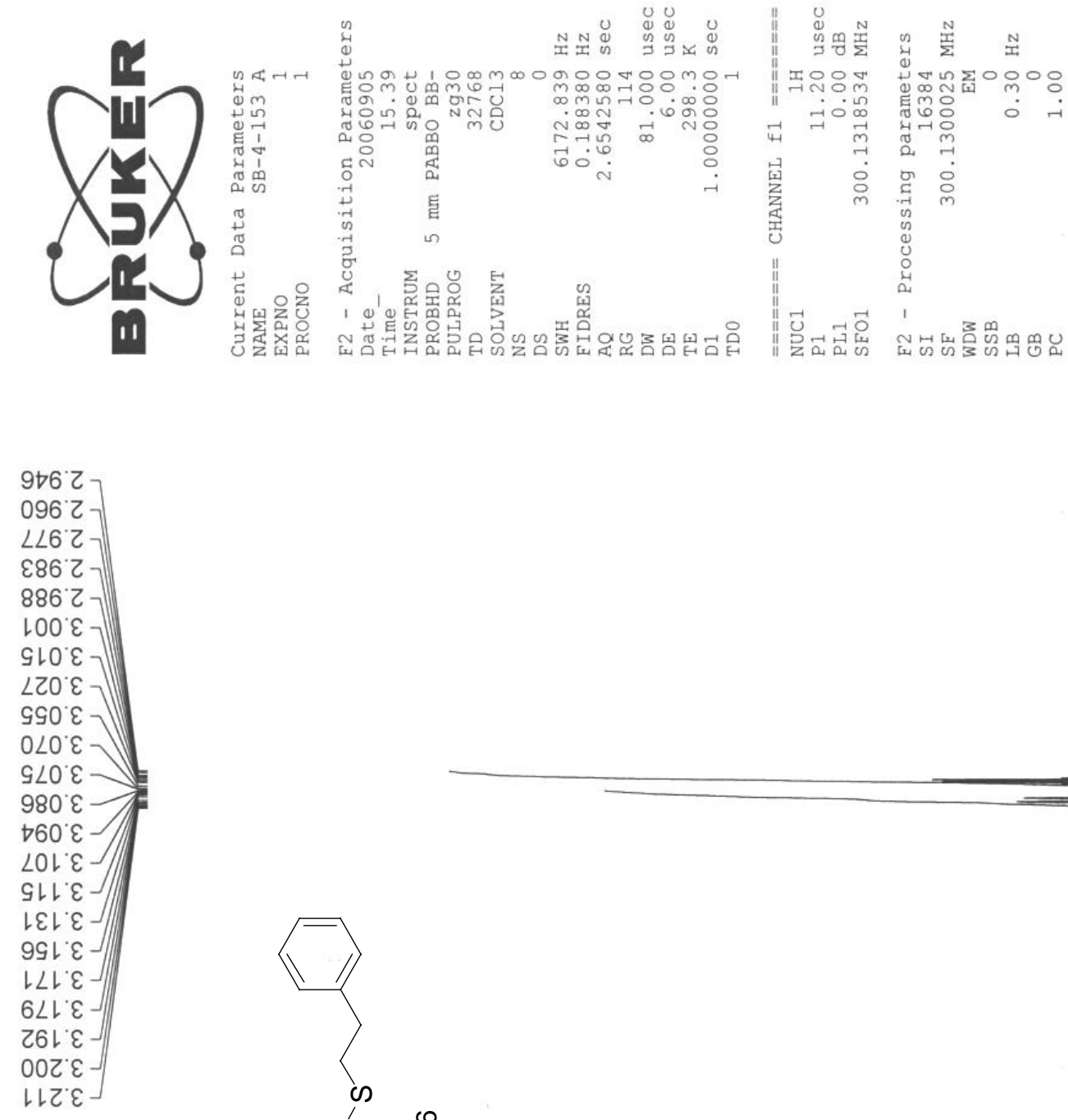

$9 Z Z^{\circ} L$
$8 \nabla Z L$
$Z 9 Z L$
$L \angle Z L$
$\varepsilon 8 Z L$
$L Z \varepsilon^{\prime} L$
$\nabla \nabla \varepsilon^{\prime} L$
$\angle 9 \varepsilon^{\circ} L$
$L \angle \varepsilon^{\prime} L$

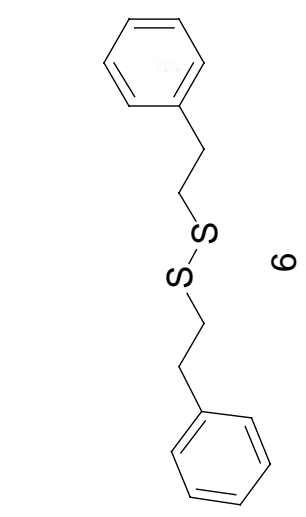



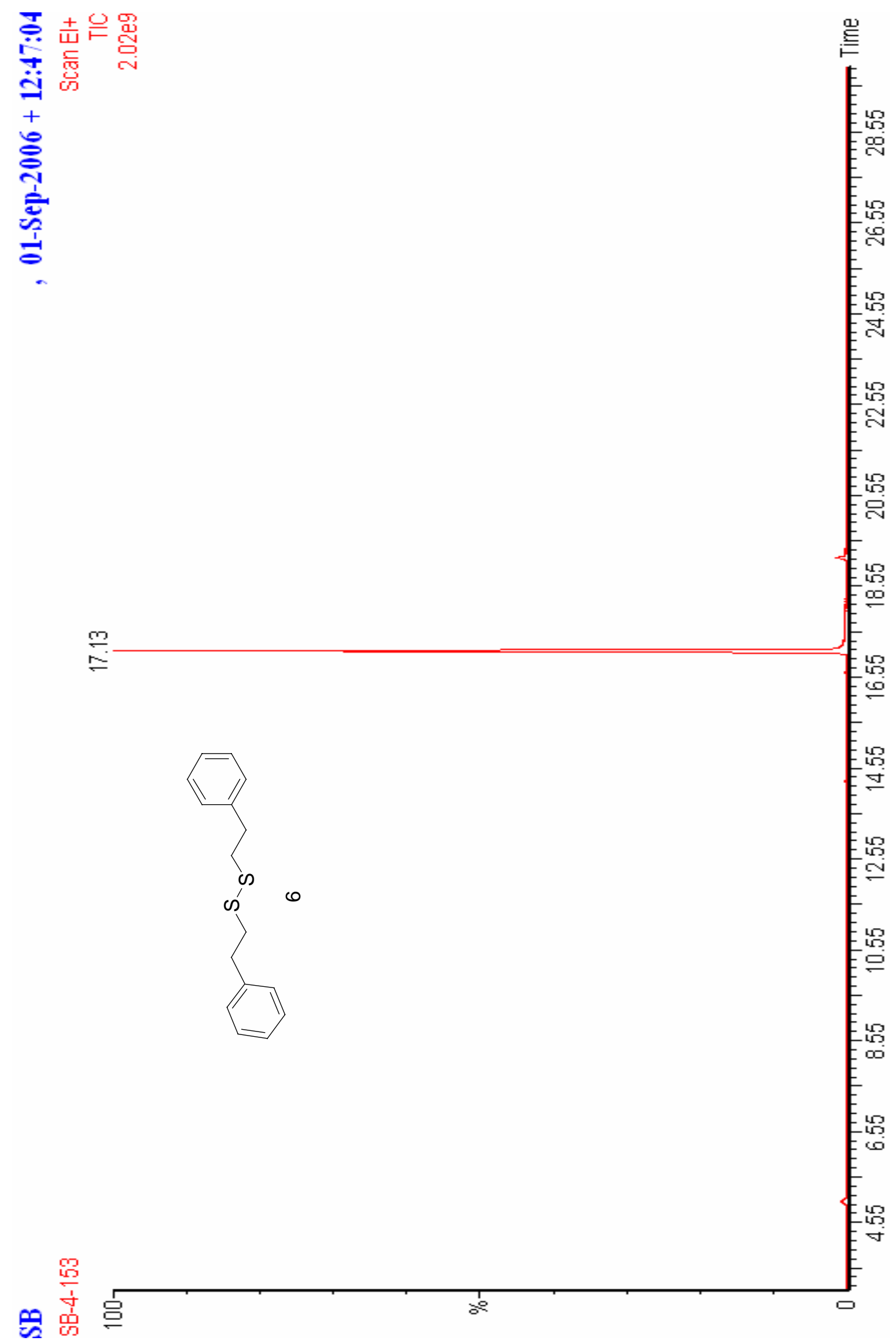

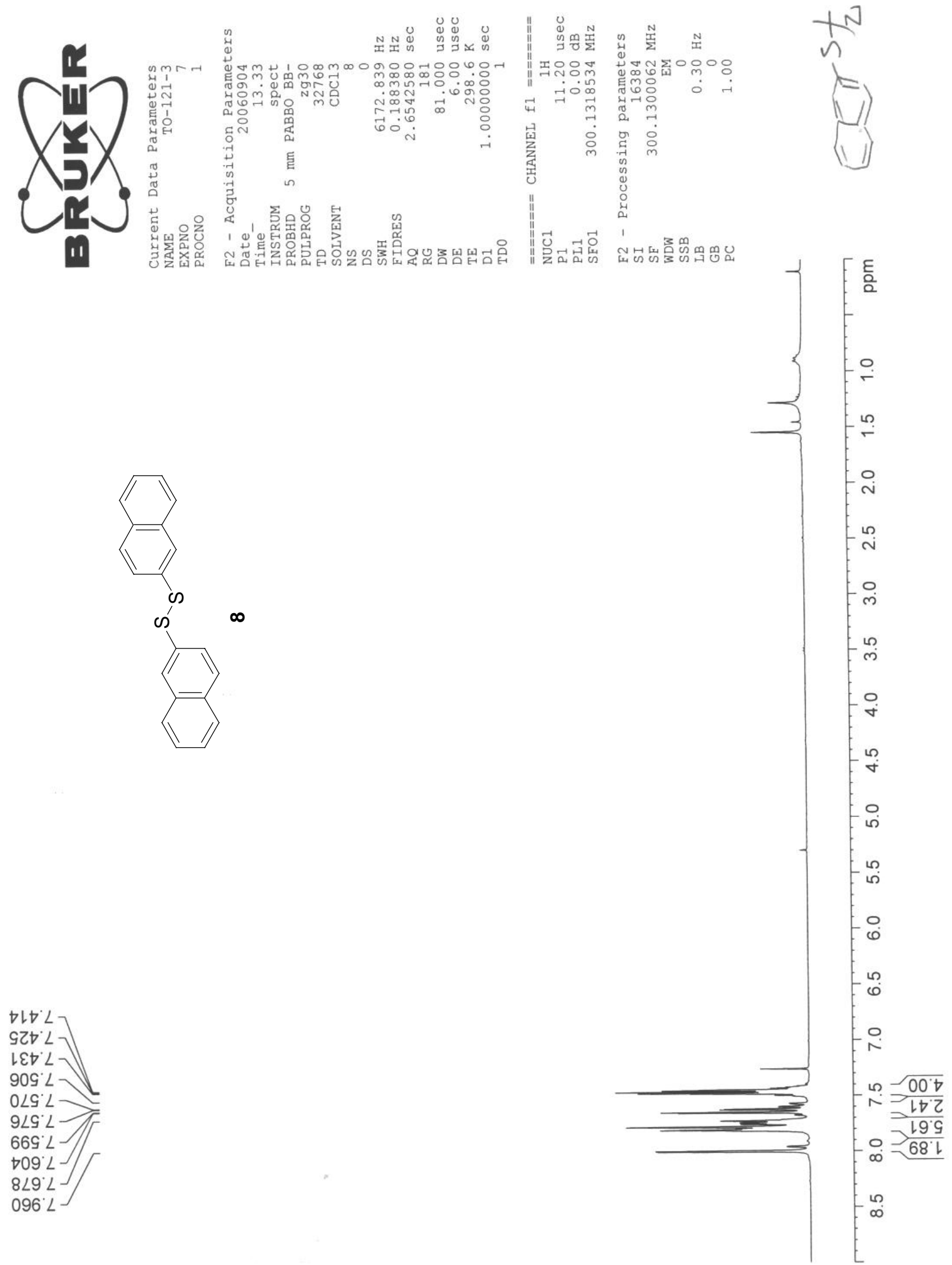

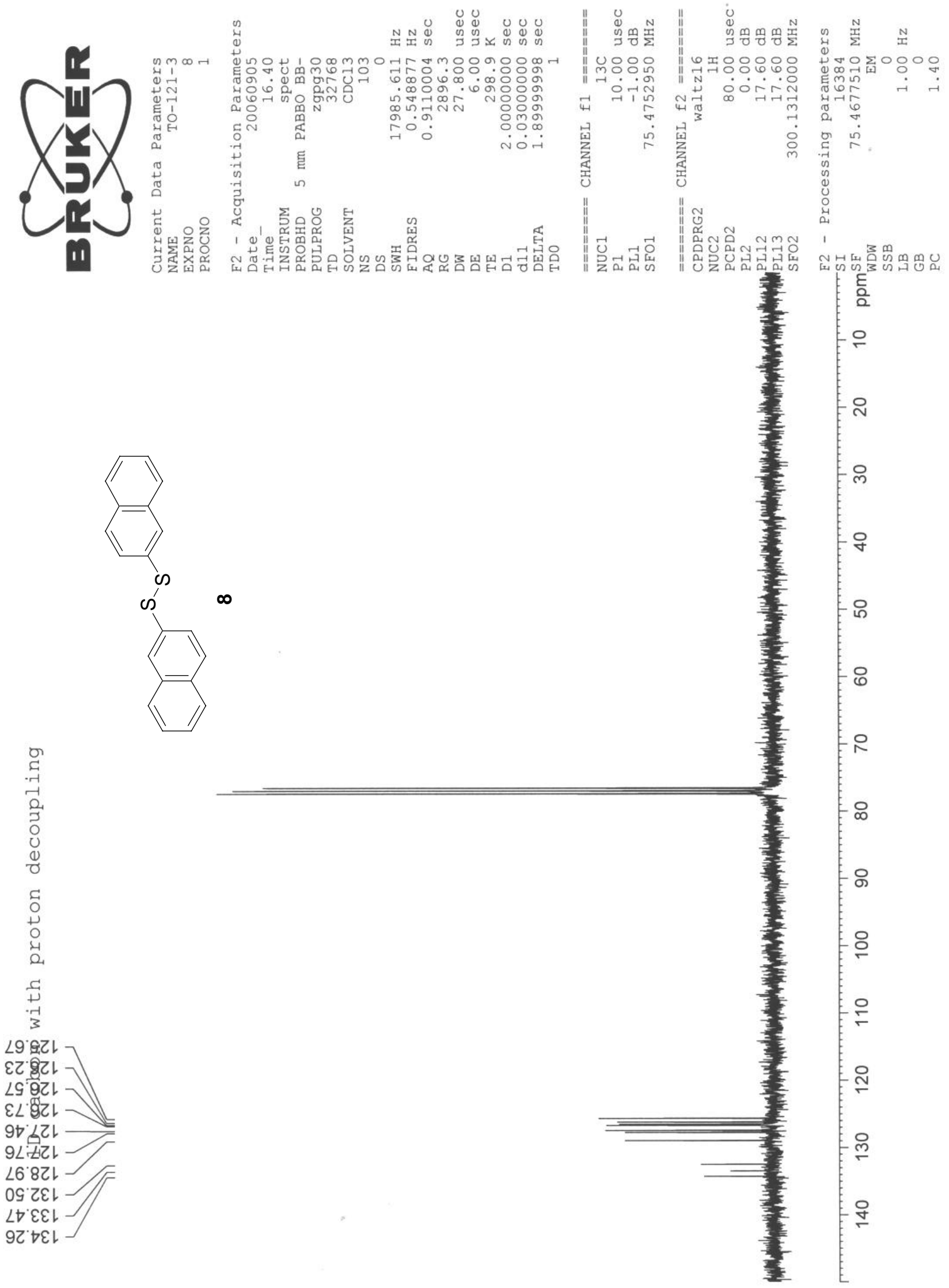

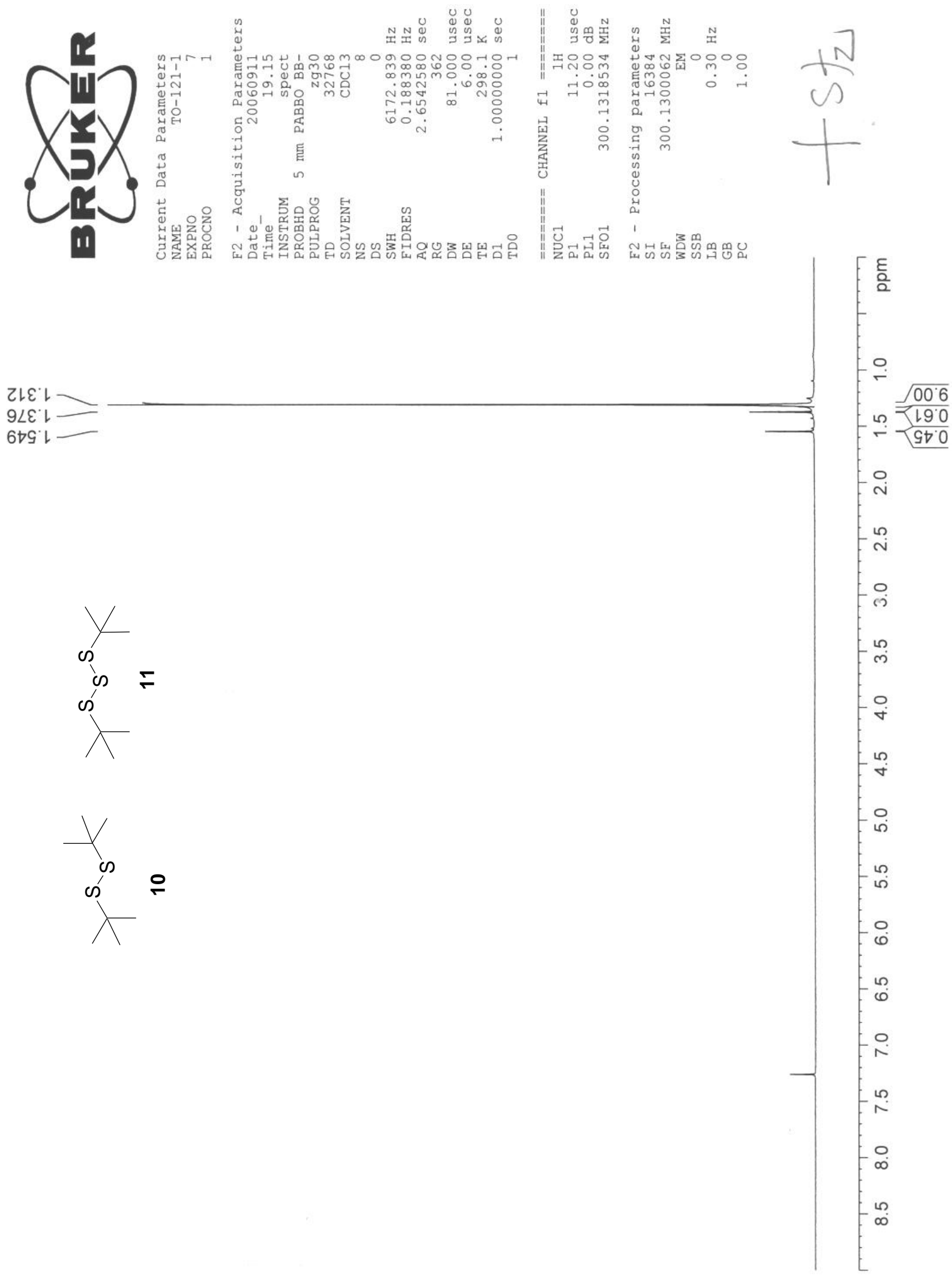


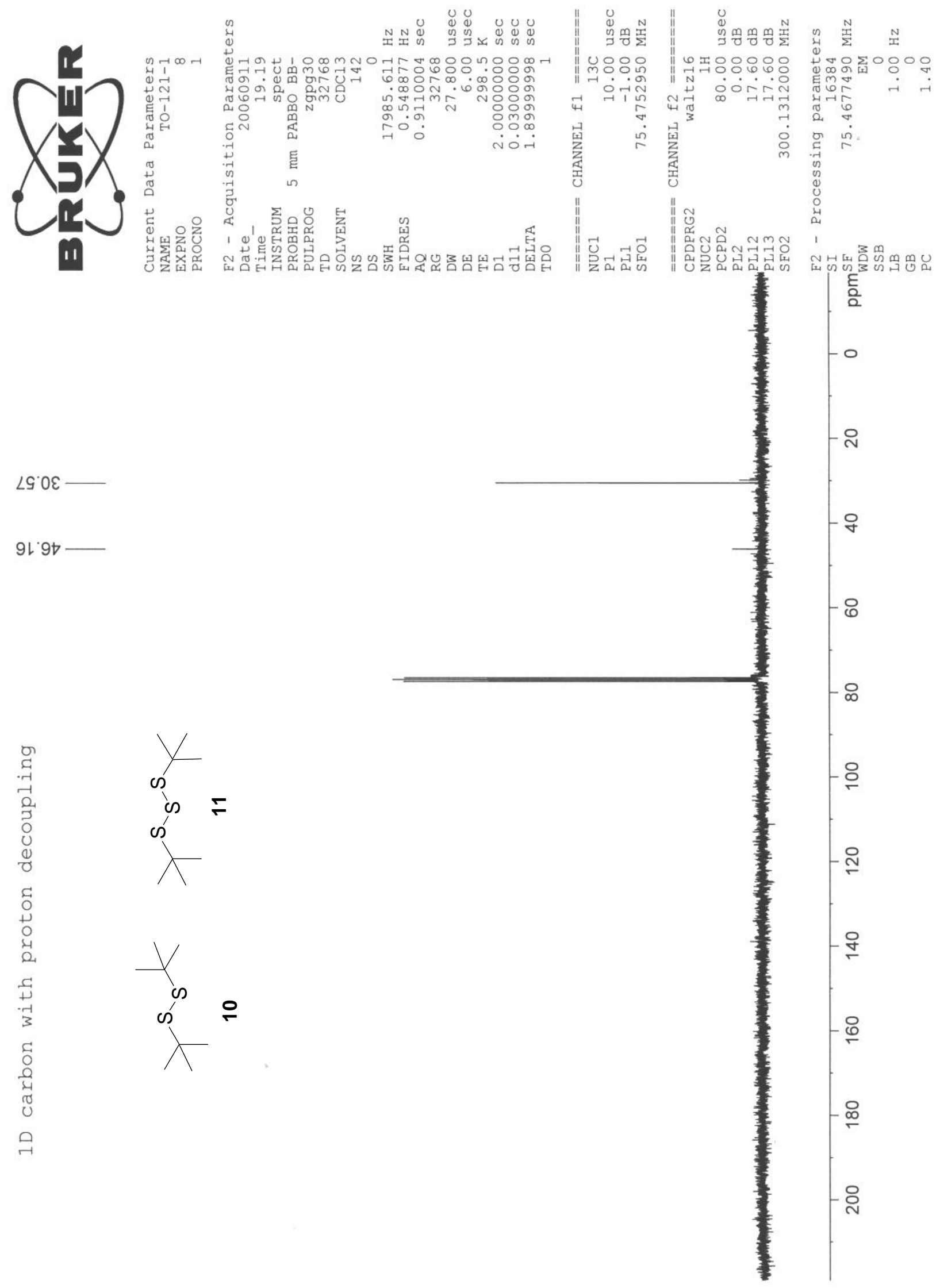



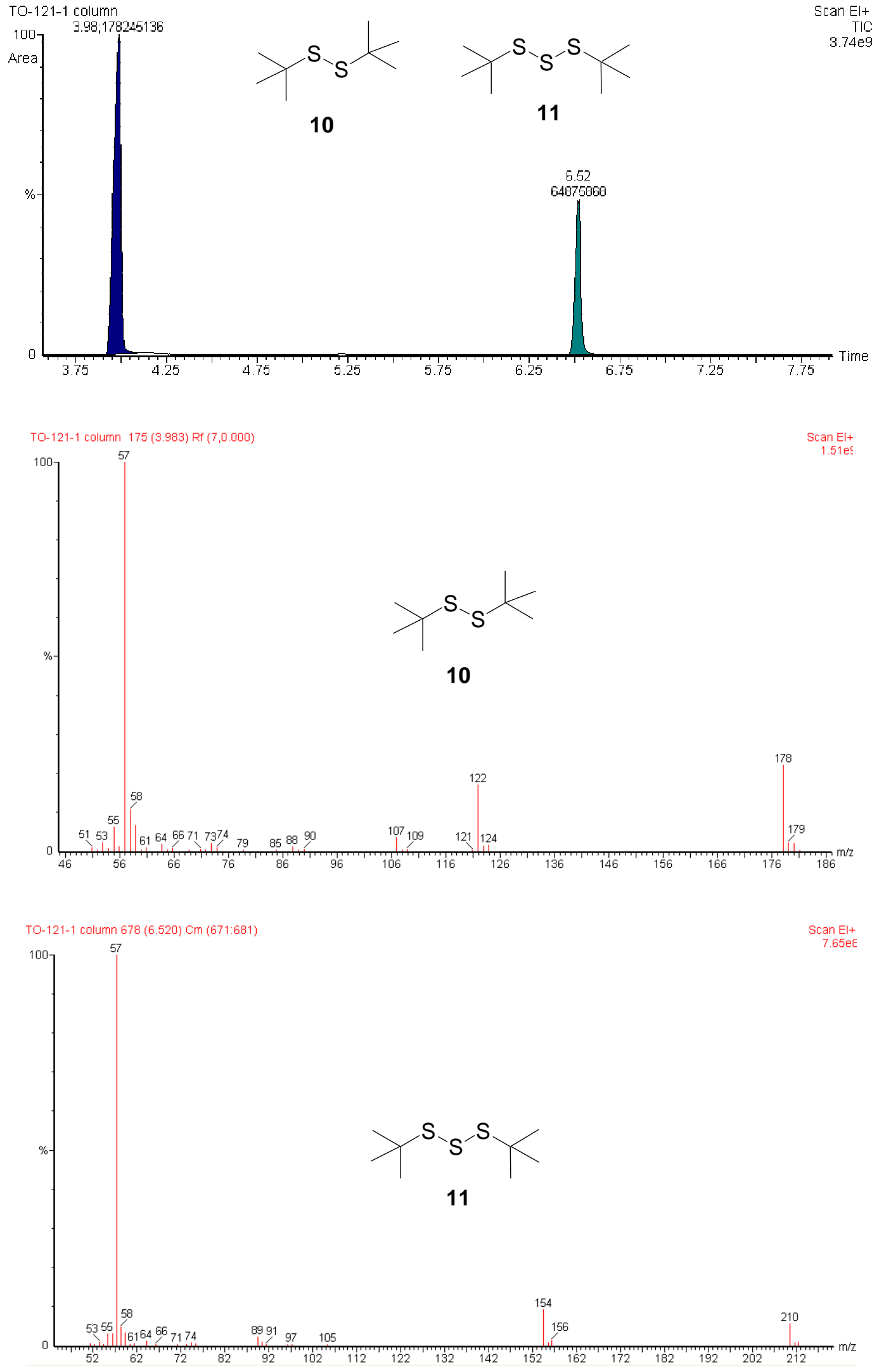

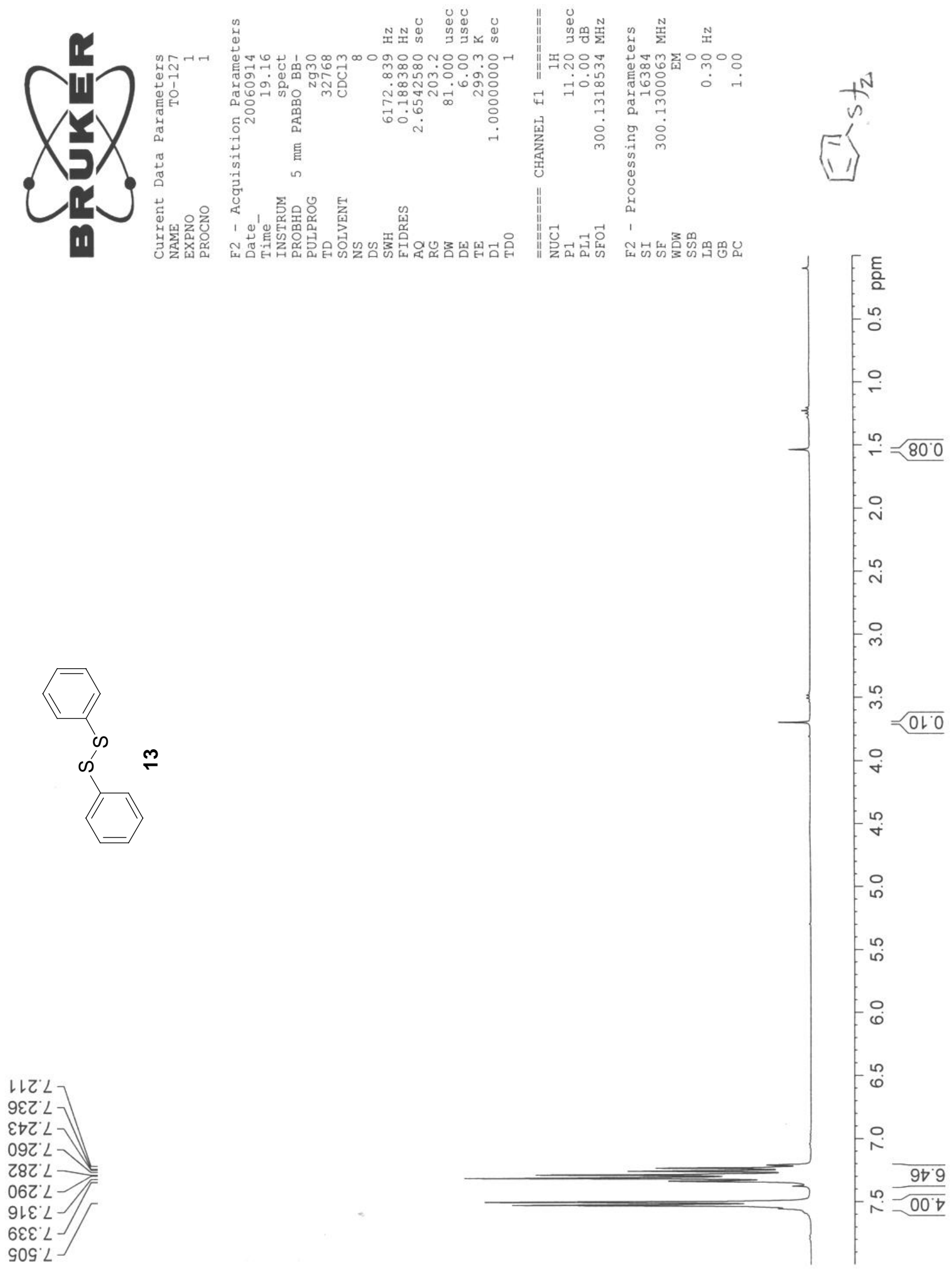

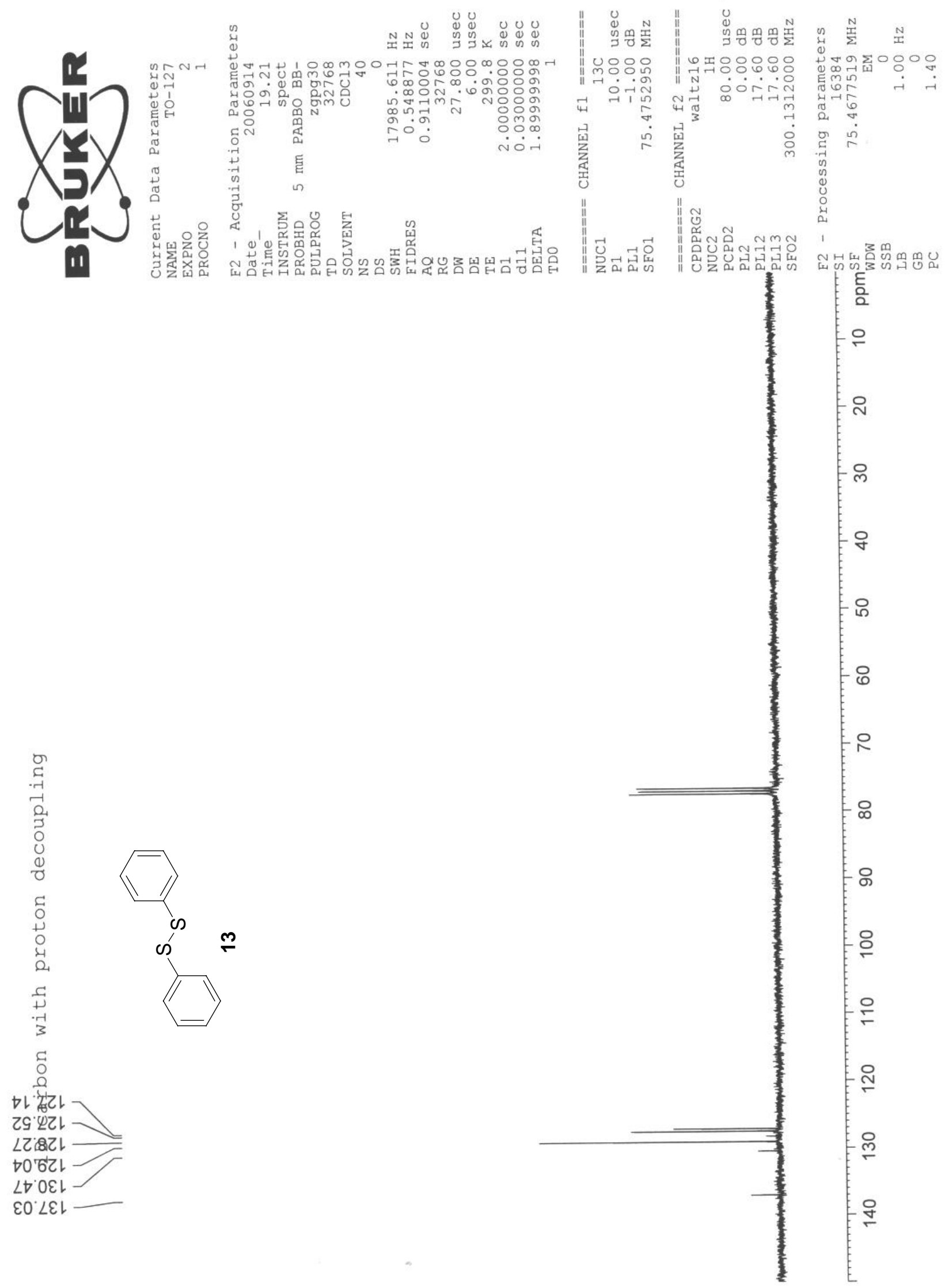

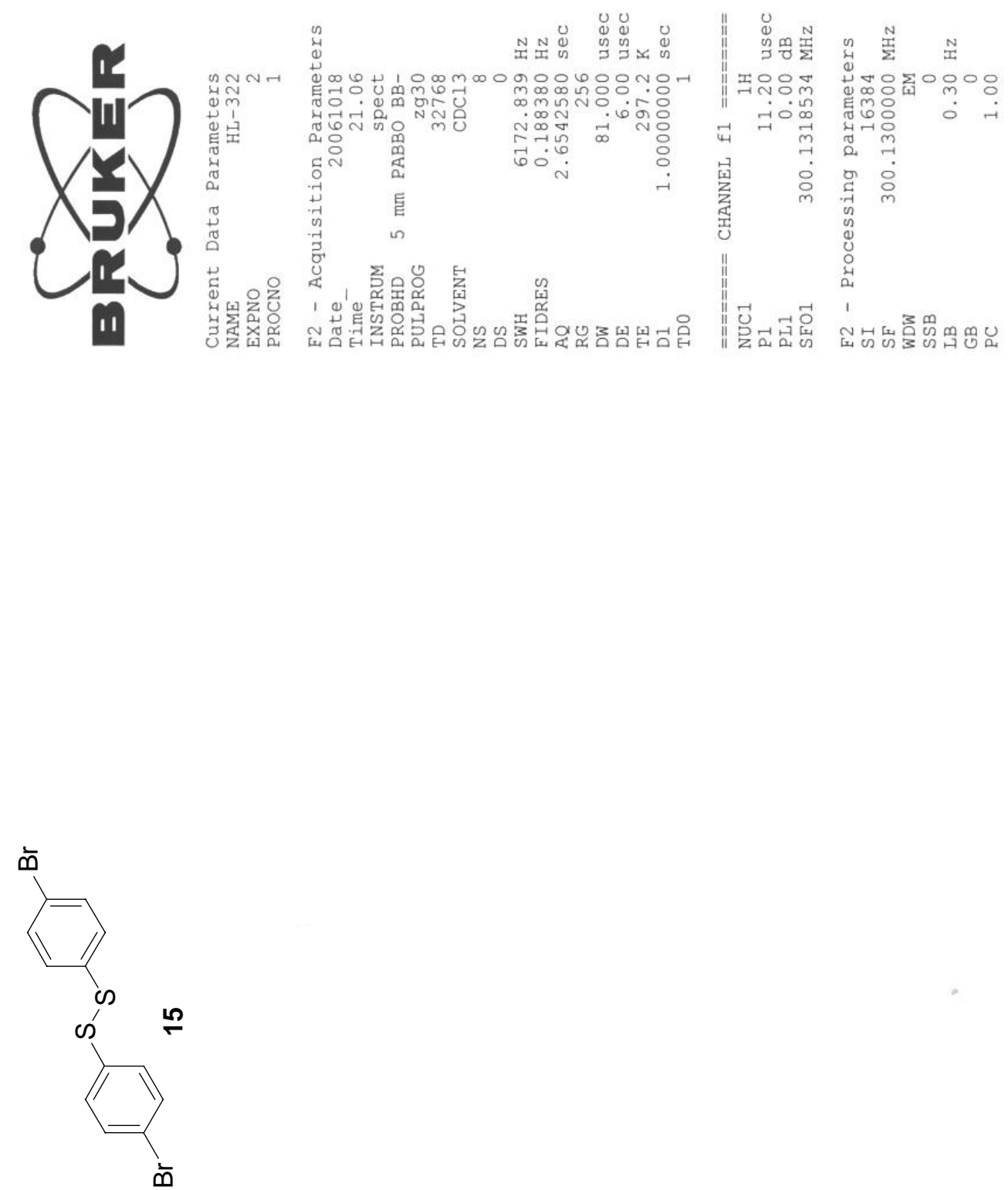

$\varepsilon \nabla \varepsilon^{\prime} L$

$L \angle E^{\prime} L$

9Et $L$

$\nabla 9 \nabla L$

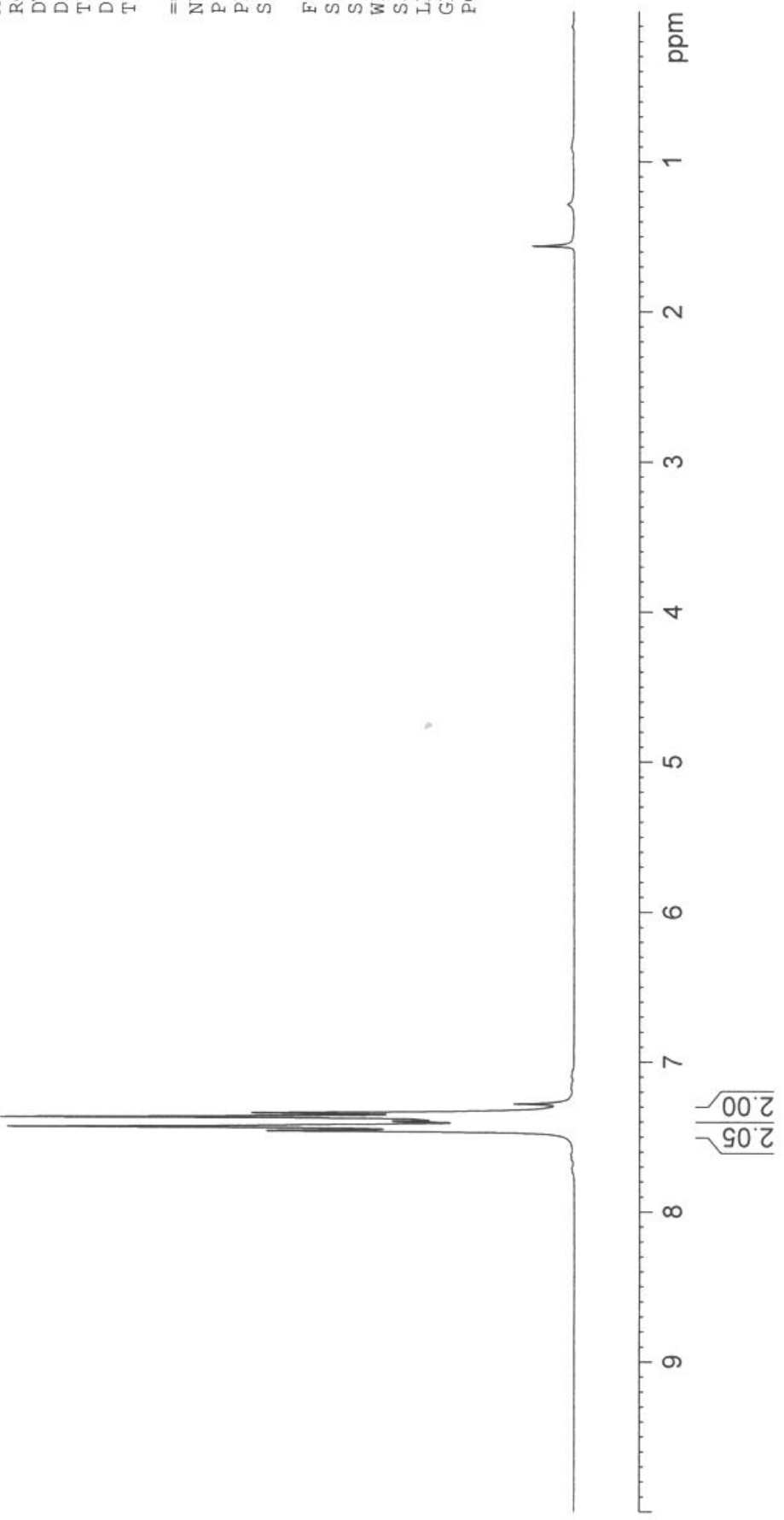



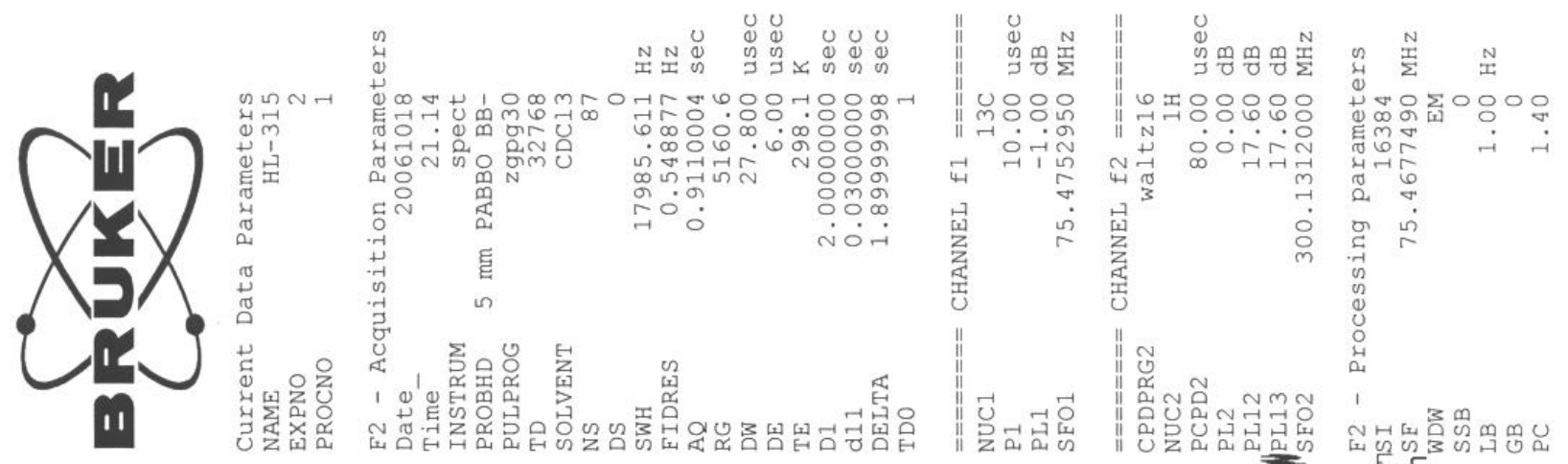

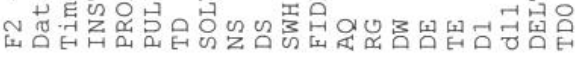
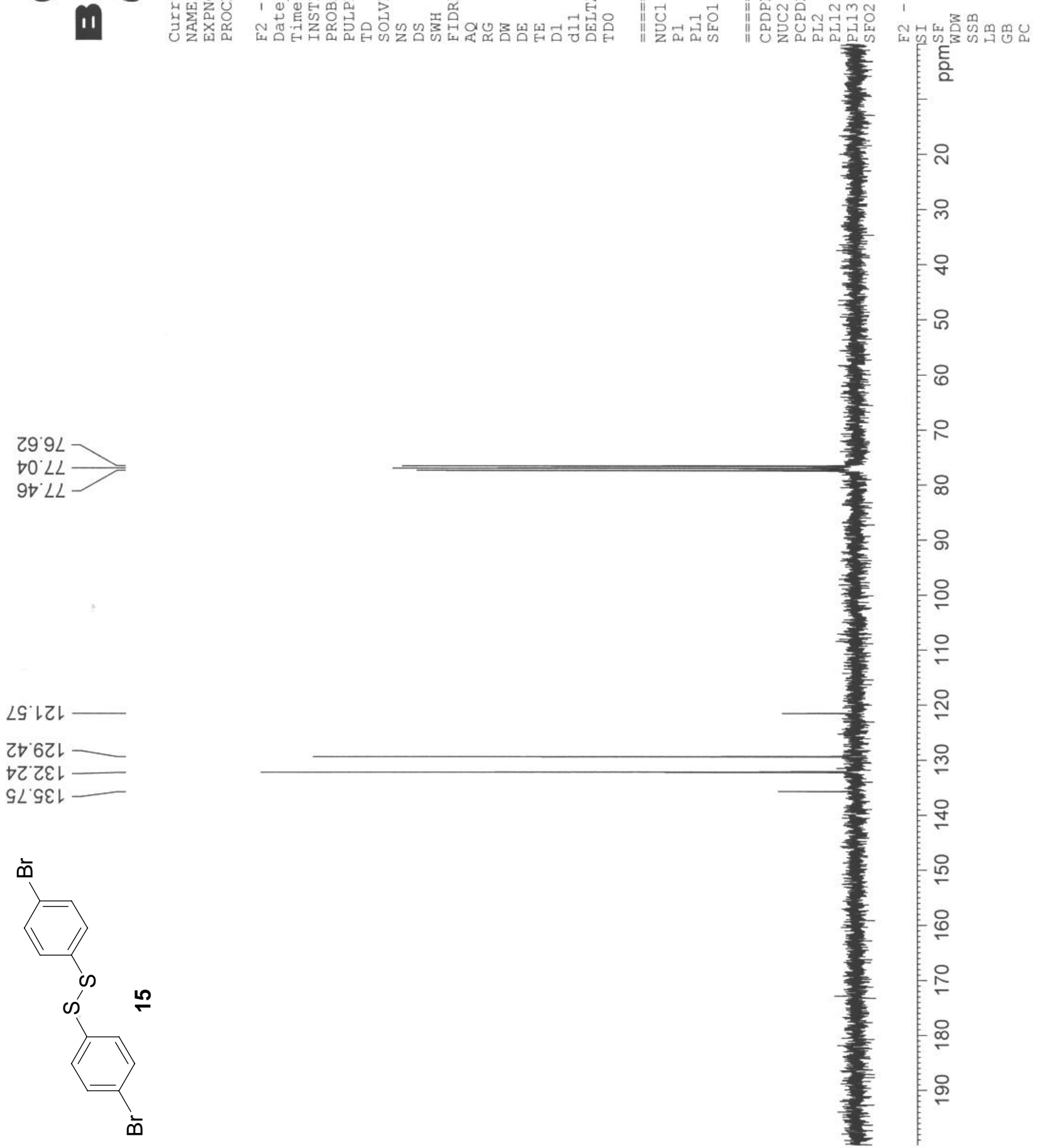

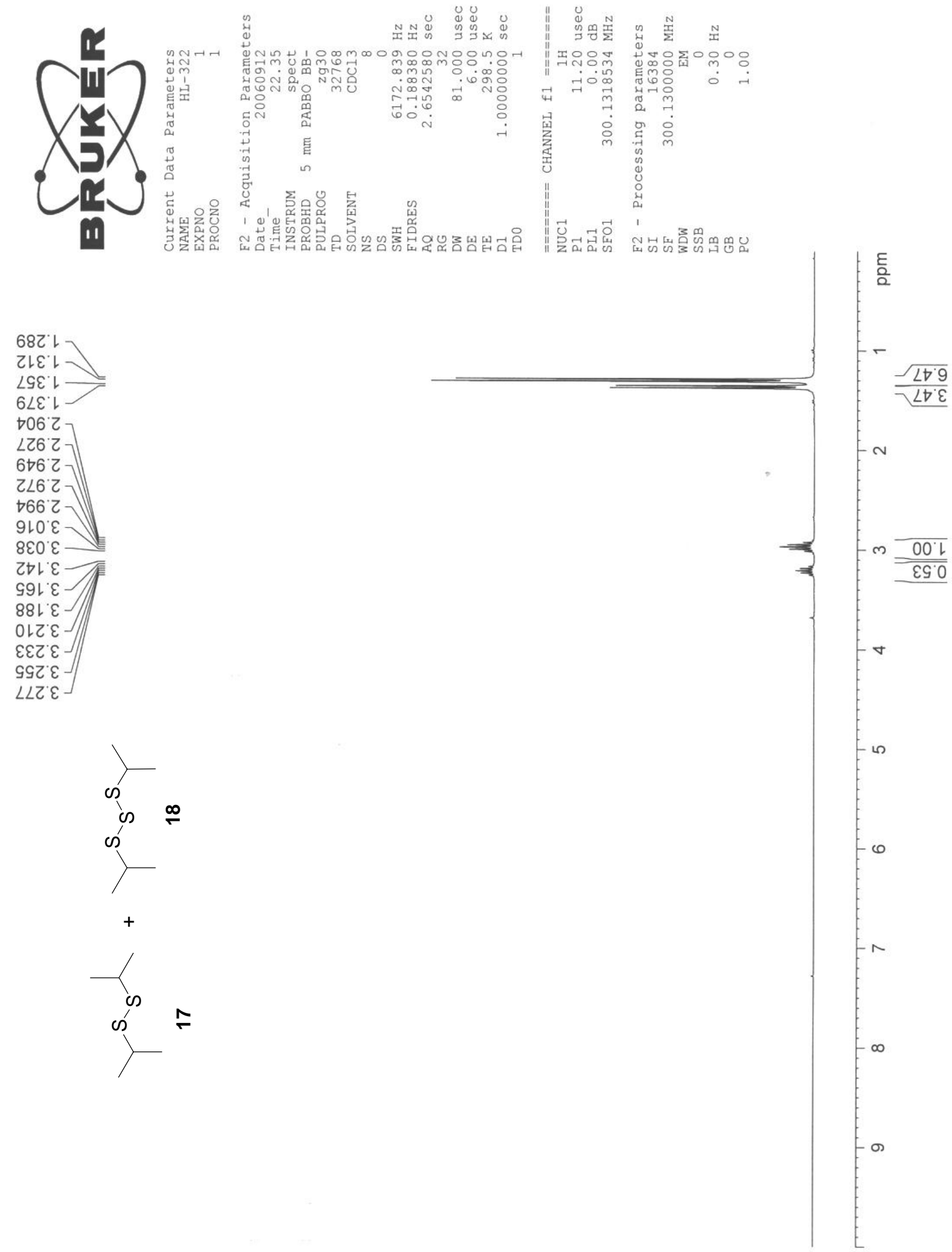

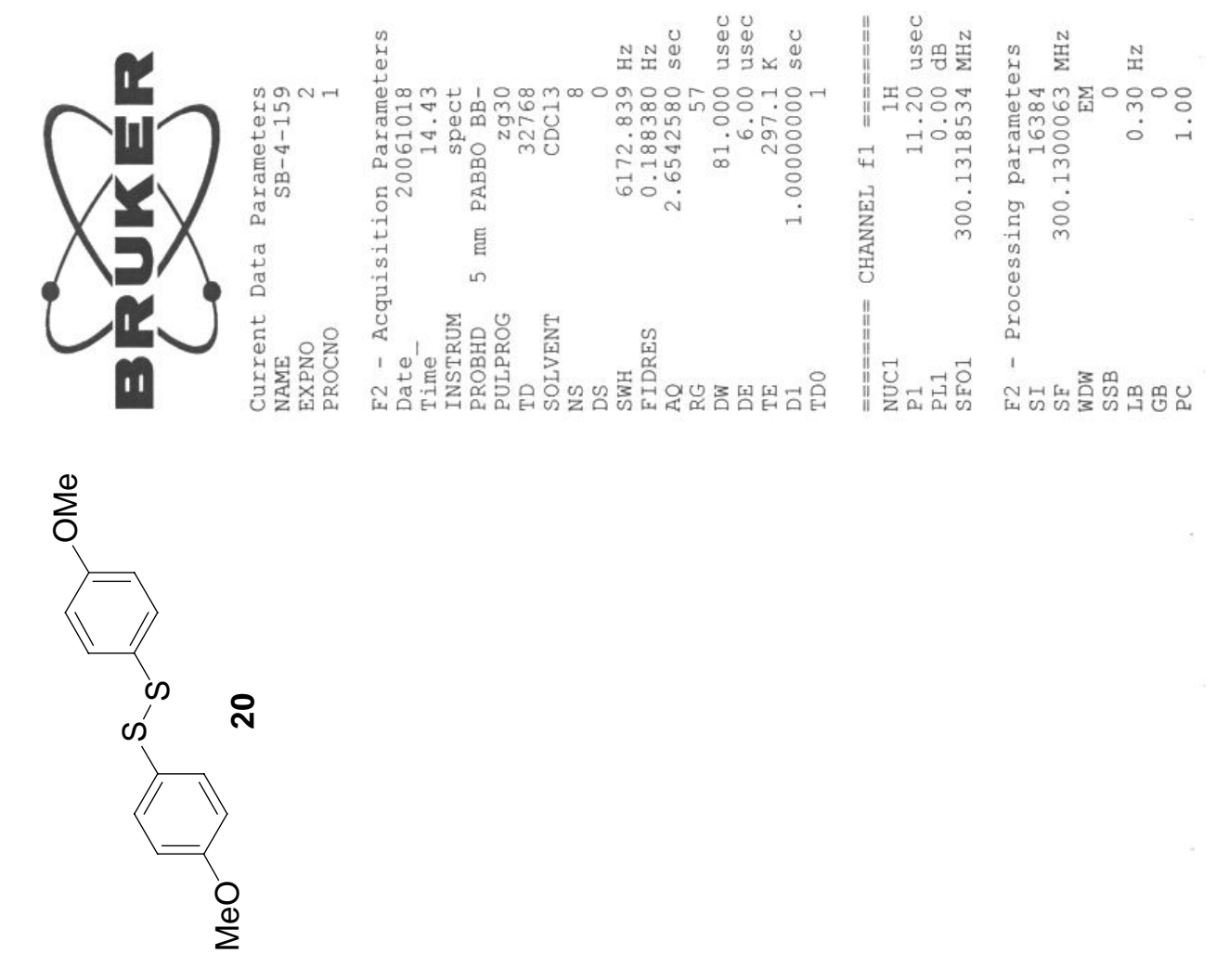

Z6L $\varepsilon-$
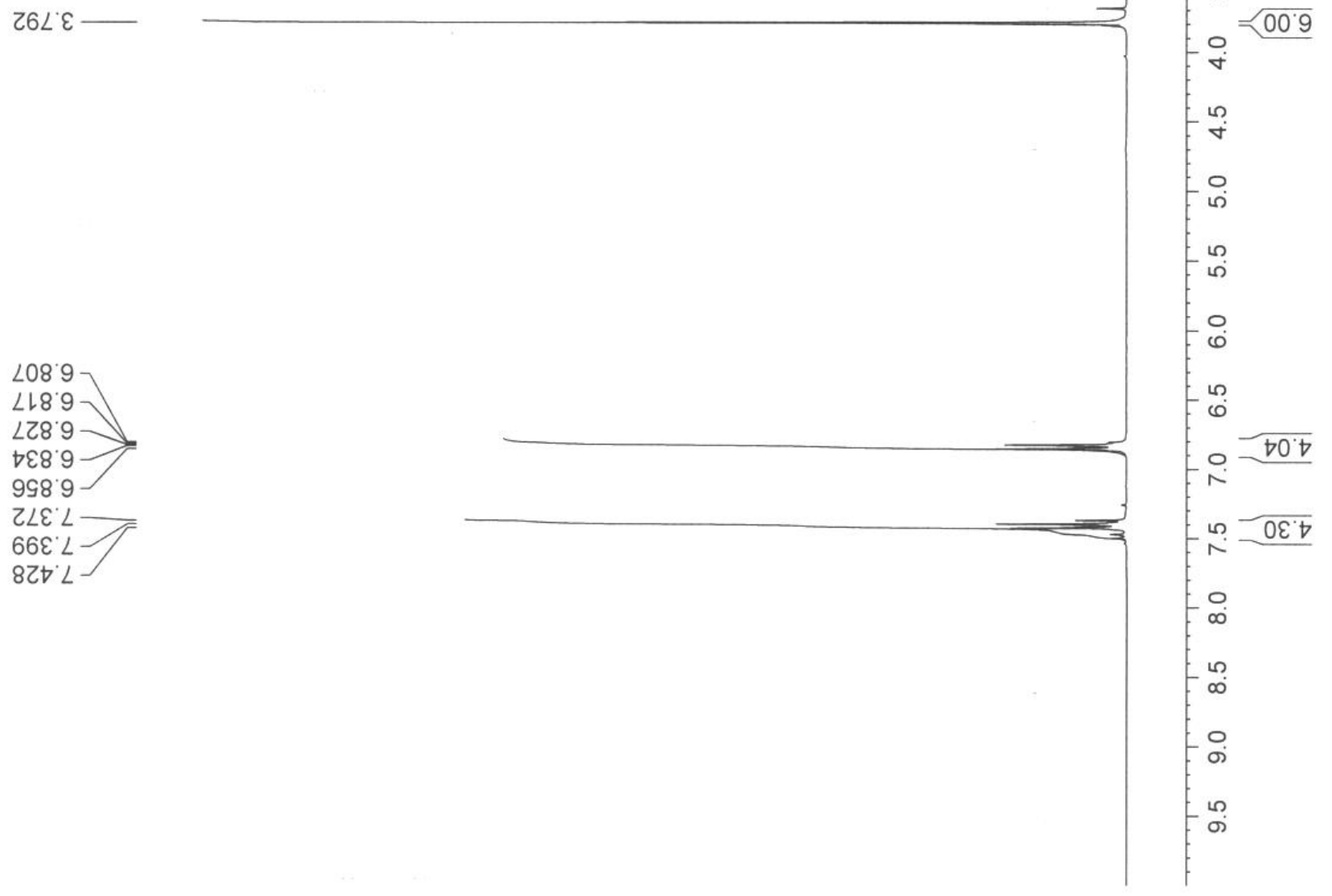


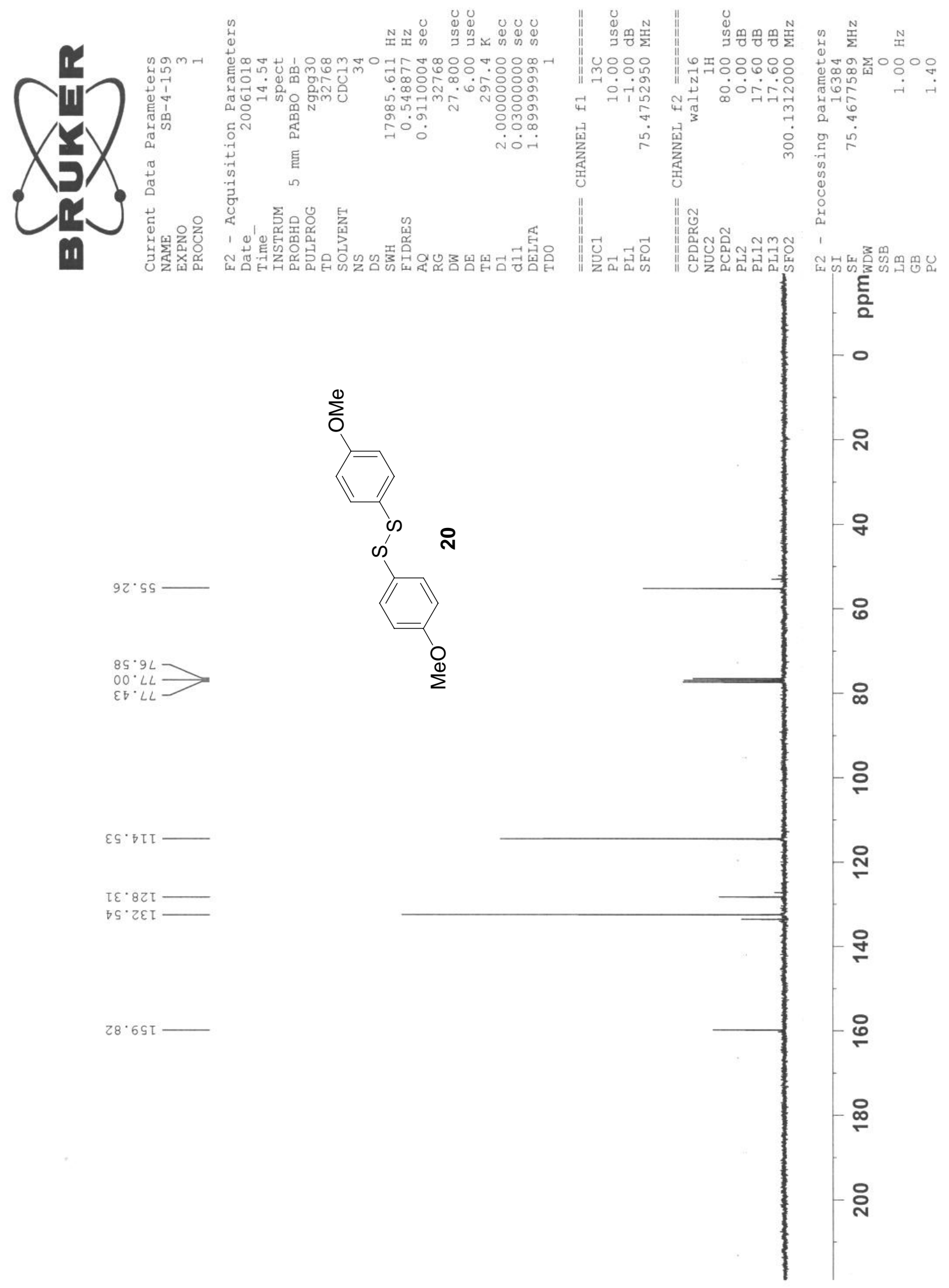



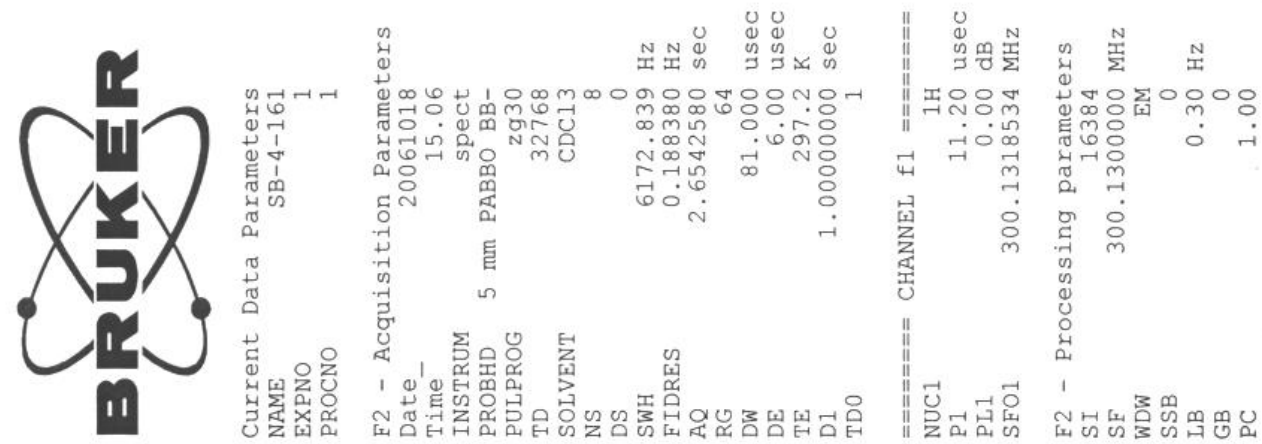

$199^{\circ} \mathcal{-}$

$\varepsilon 80^{\circ} \supset$

$06 Z^{\circ} \mathrm{L}$

OLE'L]

$6 乙 \varepsilon^{\prime} L$

S†E'L

ISE' $L$

เ9E' $L$

ZLE' $L$

$98 \varepsilon^{\circ} L$

$\angle O T L$

จレレ

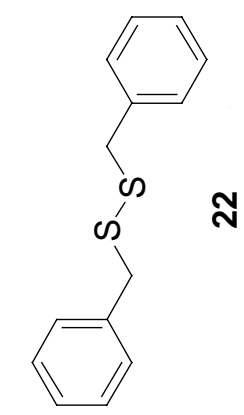

N
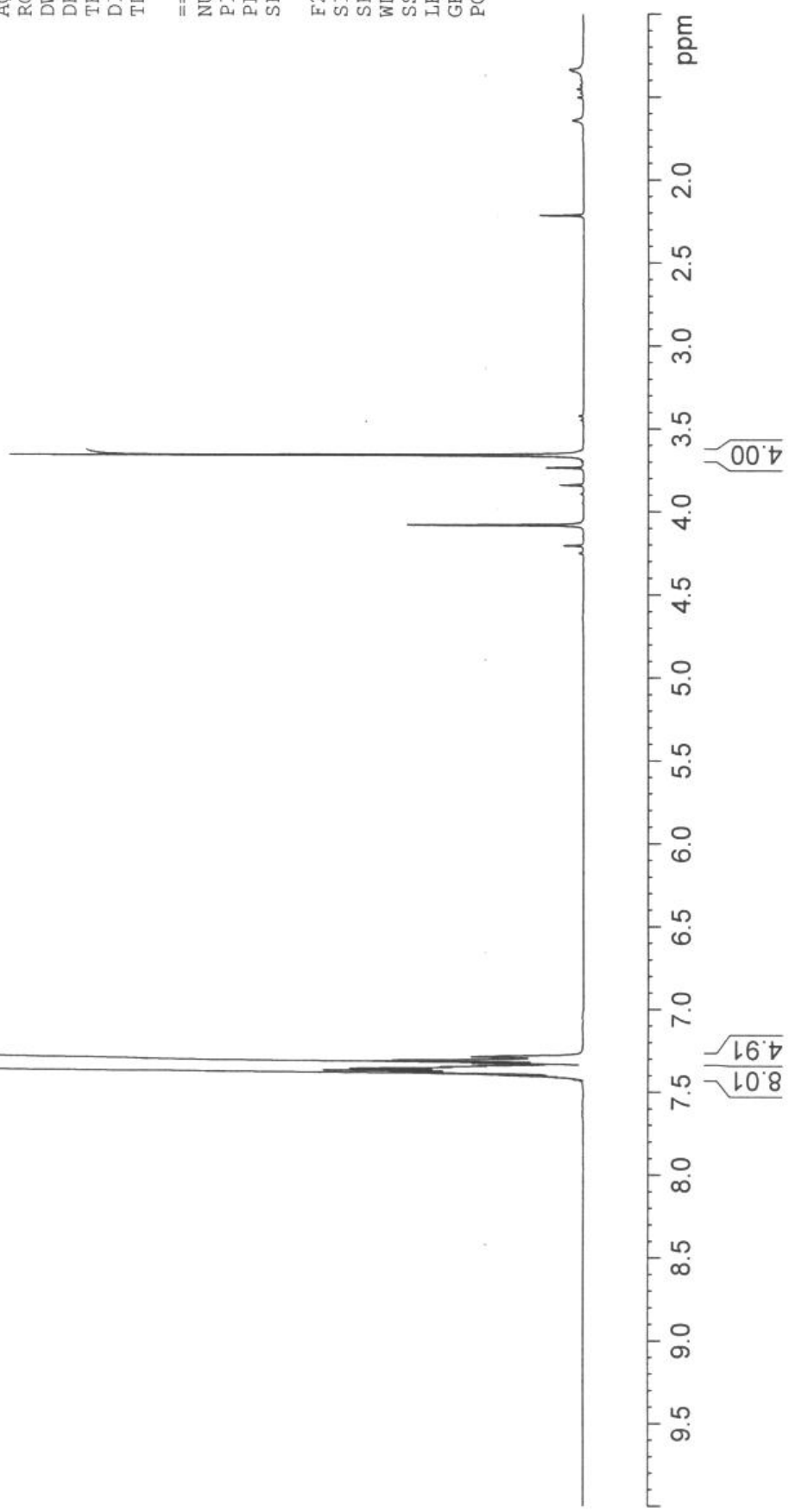


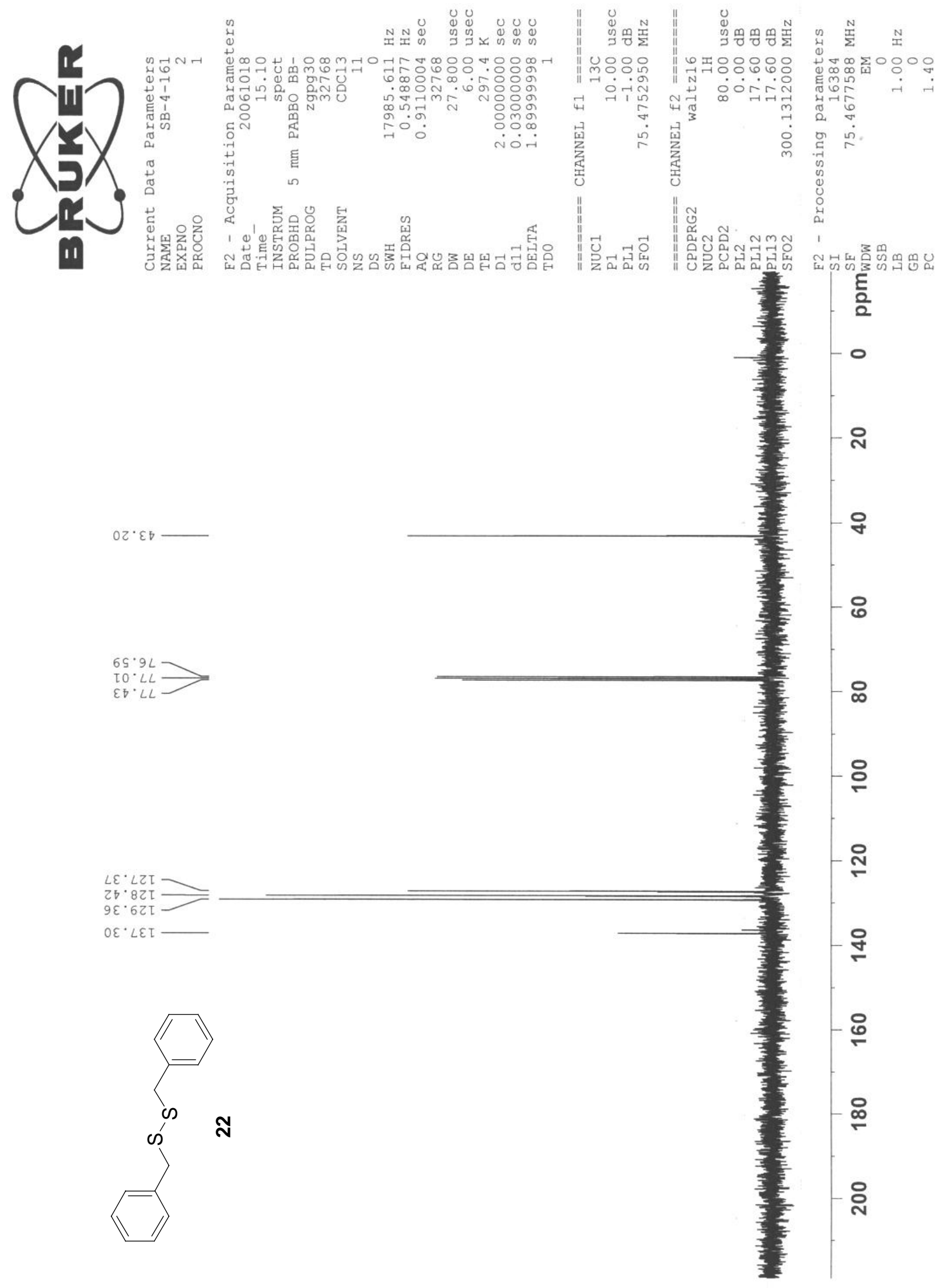



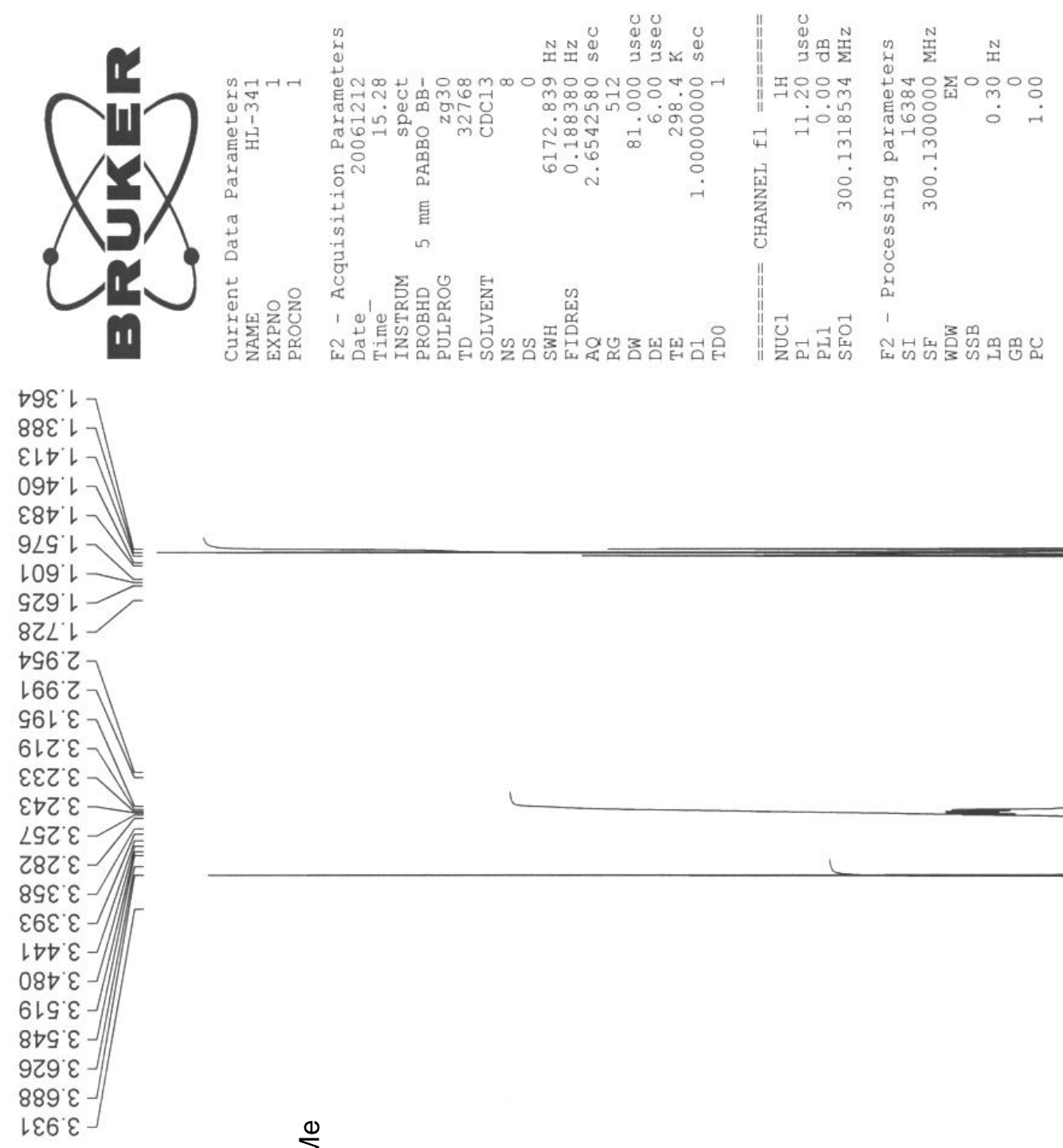

เE6 $\varepsilon$

عoz 6

เZヤ 6

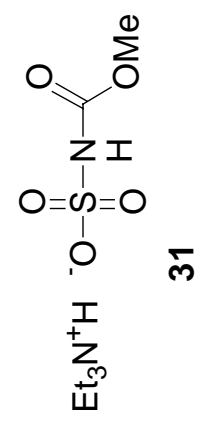




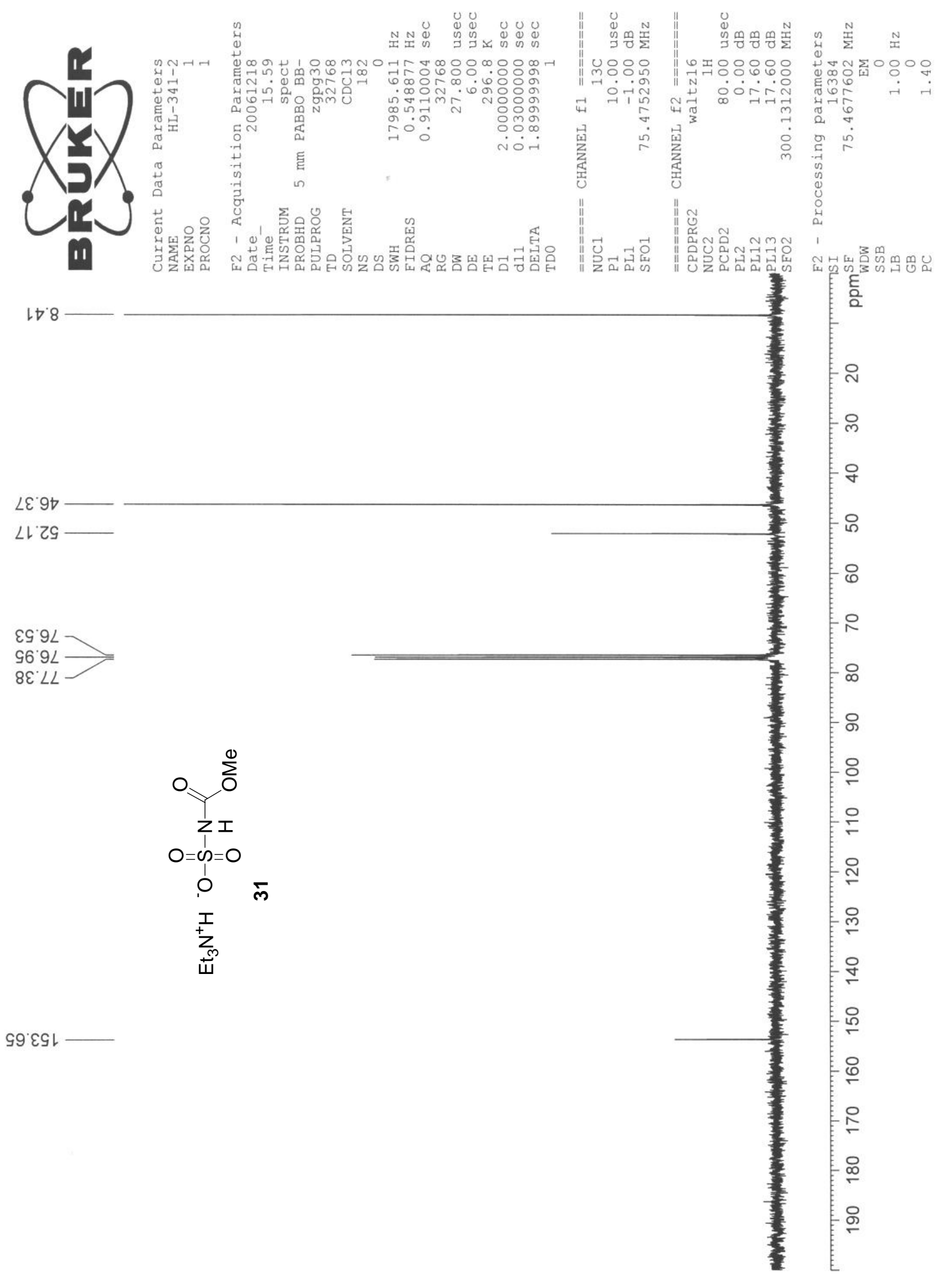

